Louisiana State University

LSU Digital Commons

$12-1-2019$

\title{
An Analysis of the Shapes of Interstellar Extinction Curves. VII. Milky Way Spectrophotometric Optical-through-ultraviolet Extinction and Its R-dependence
}

\author{
E. L. Fitzpatrick \\ Villanova University \\ Derck Massa \\ Space Science Institute \\ Karl D. Gordon \\ Space Telescope Science Institute \\ Ralph Bohlin \\ Space Telescope Science Institute \\ Geoffrey C. Clayton \\ Louisiana State University
}

Follow this and additional works at: https://digitalcommons.Isu.edu/physics_astronomy_pubs

\section{Recommended Citation}

Fitzpatrick, E., Massa, D., Gordon, K., Bohlin, R., \& Clayton, G. (2019). An Analysis of the Shapes of Interstellar Extinction Curves. VII. Milky Way Spectrophotometric Optical-through-ultraviolet Extinction and Its R-dependence. Astrophysical Journal, 886 (2) https://doi.org/10.3847/1538-4357/ab4c3a 


\title{
An Analysis of the Shapes of Interstellar Extinction Curves. VII. Milky Way Spectrophotometric Optical-through-ultraviolet Extinction and Its $\boldsymbol{R}$-dependence ${ }^{*}$
}

\author{
E. L. Fitzpatrick ${ }^{1}$ (1) Derck Massa $^{2}$ (i), Karl D. Gordon ${ }^{3,4}$ (1) Ralph Bohlin $^{3}$ (1), and Geoffrey C. Clayton ${ }^{5}$ (D) \\ ${ }^{1}$ Department of Astrophysics \& Planetary Science, Villanova University, 800 Lancaster Avenue, Villanova, PA 19085, USA \\ ${ }^{2}$ Space Science Institute 4750 Walnut Street, Suite 205 Boulder, CO 80301, USA; dmassa@ spacescience.org \\ ${ }^{3}$ Space Telescope Science Institute, 3700 San Martin Drive, Baltimore, MD 21218, USA \\ ${ }^{4}$ Sterrenkundig Observatorium, Universiteit Gent, Gent, Belgium \\ ${ }^{5}$ Department of Physics \& Astronomy, Louisiana State University, Baton Rouge, LA 70803, USA \\ Received 2019 September 20; revised 2019 October 7; accepted 2019 October 8; published 2019 November 26
}

\begin{abstract}
We produce a set of 72 NIR-through-UV extinction curves by combining new Hubble Space Telescope/STIS optical spectrophotometry with existing International Ultraviolet Explorer spectrophotometry (yielding gapless coverage from 1150 to $10000 \AA$ ) and NIR photometry. These curves are used to determine a new, internally consistent NIR-through-UV Milky Way mean curve and to characterize how the shapes of the extinction curves depend on $R(V)$. We emphasize that while this dependence captures much of the curve variability, considerable variation remains that is independent of $R(V)$. We use the optical spectrophotometry to verify the presence of structure at intermediate wavelength scales in the curves. The fact that the optical-through-UV portions of the curves are sampled at relatively high resolution makes them very useful for determining how extinction affects different broadband systems, and we provide several examples. Finally, we compare our results to previous investigations.
\end{abstract}

Unified Astronomy Thesaurus concepts: Interstellar dust (836); Interstellar dust extinction (837); Interstellar dust processes (838); Interstellar absorption (831); Interstellar reddening (853); Interstellar scattering (854)

Supporting material: figure set

\section{Introduction}

Dust grains play a large number of roles in the interstellar medium (ISM), serving, for example, as the primary formation site for $\mathrm{H}_{2}$, as an important heat source (via the photoelectric effect), and as a chemical reservoir for the heavy elements. Arguably, however, their most significant effect is in modulating the flow of electromagnetic radiation through interstellar space via the combined effect of absorption and scattering. The ability of grains to transmit, redirect, and transmute electromagnetic energy is enormously important to the physics of interstellar space and, perhaps most far-reaching, places profound limits on our ability to study the universe at optical and ultraviolet (UV) wavelengths.

Studies of the interstellar "extinction curve," that is, the wavelength dependence of the combined effect of absorption and scattering out of the line of sight, have shown it to be highly spatially variable. This poses significant problems because a detailed knowledge of the curve is essential in numerous astrophysical applications, such as reconstructing the intrinsic spectral energy distributions (SEDs) of objects ranging from nearby stars to the most distant galaxies or for understanding the deposition of electromagnetic energy in starforming regions. On the other hand, these variations are a boon for the study of the dust itself, because they reflect general differences in the grain populations from sight line to sight line. Understanding how the various spectral regions of the curves relate to each other and how they respond to changes in the interstellar environments can provide information critical for

\footnotetext{
* Based on observations made with the NASA/ESA Hubble Space Telescope, obtained at the Space Telescope Science Institute, which is operated by the Association of Universities for Research in Astronomy, Inc., under NASA contract NAS5-26555. These observations are associated with program 13760.
}

characterizing the size, composition, and structure of interstellar grains (e.g., Weingartner \& Draine 2001; Clayton et al. 2003; Zubko et al. 2004).

Detailed investigations of the wavelength dependence of interstellar extinction began with measurements in the optical, generally based on broadband photometry. Early on, it was discovered that optical extinction curves may vary in shape from sight line to sight line, as exemplified by the $2.2 \mu \mathrm{m}^{-1}$ "knee" reported by Whitford (1958). Higher resolution studies revealed the presence of the "Very Broad Structure," first reported by Whiteoak (1966) and studied in detail by York (1971). This structure is apparent in the "continuum subtracted" residuals presented most recently by Maíz Apellániz et al. (2014). Equally early, it was recognized that some of these shape variations were related to the ratio of total-to-selective extinction, $R(V) \equiv A(V) / E(B-V)$ (Johnson \& Borgman $1963)$. The $R(V)$-dependence of optical and, also, UV extinction was later quantified by Cardelli et al. (1989), and it has become standard practice to represent extinction curves as a family whose broad characteristics are dependent on $R(V)$ (Fitzpatrick 1999, 2004; Valencic et al. 2004; Gordon et al. 2009; Maíz Apellániz et al. 2014).

It is somewhat remarkable that-despite its observational accessibility-the optical is not the best-characterized region of the interstellar extinction curve. The difficulty in obtaining ground-based spectrophotometry and the resultant paucity of spectroscopic resolution studies of optical extinction have prevented detailed investigations of the optical extinction features, their spatial variability, and their relationship to features at other wavelengths. Remarkably, is it the UV, accessible only by space-based instruments, where extinction has been most extensively studied at spectroscopic resolution (Witt et al. 1984; Fitzpatrick \& Massa 1986, 1988, 1990; Aiello et al. 1988; 
Cardelli et al. 1989; Valencic et al. 2004; Gordon et al. 2009), primarily as a result of the large database of UV spectrophotometry obtained by the International Ultraviolet Explorer satellite (IUE).

To fill in this gap in our knowledge of optical extinction, and to better characterize the relationship between extinction in the optical and that at other wavelengths, we initiated a Hubble Space Telescope (HST) SNAP program using the lowresolution STIS optical and near-infrared (NIR) gratings to observe a carefully selected set of moderately reddened earlytype stars that were previously observed in the UV. These observations provide low-resolution spectrophotometry covering the spectral range from the NIR to the near-UV region, that is, between $\sim 1 \mu \mathrm{m}$ and $\sim 3000 \mathrm{~A}$. When coupled with the existing UV data, these yield well-calibrated, low-resolution SEDs for the program stars from 1150 to $10000 \AA$, which can be used to construct detailed extinction curves. These data resolve most, if not all, structure in the curves shortward of $1 \mu \mathrm{m}$; characterize the detailed shapes of the extinction curves in largely unexplored spectral regions; reveal the degree of variability in NIR through near-UV curves; and allow us to relate this variability to that seen in the UV and infrared.

The current paper utilizes IUE and STIS low-resolution spectrophotometry along with broadband NIR photometry from the Two Micron All-Sky Survey (2MASS) to study the $R(V)$-dependence of extinction along lines of sight sampling a range of Milky Way environments. A detailed analysis and characterization of the structure observed in optical extinction curves is addressed in a companion paper (Massa et al. 2020).

Section 2 describes the selection of sight lines used in our analysis, as well as the processing of the STIS, IUE, and 2MASS data. Section 3 explains how the extinction curves were derived. Section 4 presents our results along with an $R(V)=3.1$ curve (which is typical of the diffuse Milky Way environment) and an $R(V)$ parameterization that captures much of the sight line-to-sight line variation. Section 5 discusses our results and compares them to previous work. Finally, Section 6 summarizes our findings.

\section{Observations and Data Processing}

\subsection{The Sample}

Our selection of program stars is based on the HST SNAP Program 13760. The initial sample included 130 reddened, normal, Milky Way, near-main-sequence (luminosity class IIIV) O7-B9.5 stars. The techniques we use to determine extinction curves give excellent results for stars in this range (see Section 3.2). This sample was restricted to stars with existing UV spectrophotometry from IUE and NIR broadband photometry from the 2MASS project and comprised two overlapping subgroups: (1) stars with moderate to high extinction $(E(B-V) \gtrsim 0.20 \mathrm{mag})$, which sample sight lines with extreme values of $R(V)$ or interesting Milky Way environments, and (2) stars that sample dust within Milky Way open clusters and star-forming regions that have the potential to sample small-scale structure in the dust distribution. Examples of the first group can be found in Valencic et al. (2004) and Fitzpatrick \& Massa (2007, hereafter F07), who summarize most of the previous IUE work, including the Clayton et al. (2000) data on low-density sight lines. The second group includes stars in open clusters observed by Aiello et al. (1982), Panek (1983), Clayton \& Fitzpatrick (1987),
Boggs \& Bohm-Vitense (1989), Fitzpatrick \& Massa (1990), and Hackwell et al. (1991).

Of the 130 Milky Way targets submitted for our SNAP program, 77 were observed. Four stars (HD 73882, HD 99872, HD 281159, and $\mathrm{Cl}^{*} \mathrm{NGC} 457$ Pes 10) were eliminated from the sample because their STIS spectra show evidence for offset and overlapping spectra from one or more other stars lying in the dispersion direction. A fifth star, HD 164865, turned out to be a late-B supergiant, B9 Iab, and could not be well fit by the models we use to produce our extinction curves. That left the final sample of 72 stars used in this paper. These stars are listed in Table 1 along with some of their basic properties (spectral type, $\mathrm{V}$ magnitude, $E(B-V)$, distance, and galactic coordinates). Membership in a stellar cluster is indicated by the cluster name in parentheses in the first column of the table.

Figure 1 shows an example of a typical program-star SED, for the case of HD 27778 (B3 V). Prominent stellar and interstellar features are indicated beneath the SED. The sources of the data are indicated near the top of the figure. All data used in this study were obtained from the IUE satellite (UV spectrophotometry), the HST ("G430L" and "G750L" optical spectrophotometry), and the 2MASS project (NIR $J H K$ photometry). In the subsections below, we describe the acquisition, processing, and calibration of the three data sets.

\subsection{Optical Data: HST}

The optical data were all obtained as part of the HST SNAP Program 13760 and consist of STIS spectrophotometry with the G430L $(2900 \leqslant \lambda \leqslant 5700 \AA)$ and G750L $(5240 \leqslant \lambda \leqslant$ $10270 \AA$ ) first-order gratings. The total wavelength coverage is 2900-10270 $\AA$ with a resolution $\lambda / \Delta \lambda$ ranging from 530 to 1040 (e.g., Bostroem \& Proffitt 2011). The G430L observations are essential for determining the stellar parameters and had a minimum signal-to-noise ratio $(\mathrm{S} / \mathrm{N})$ goal of $20: 1$ per pixel. G750L observations are always more problematic because of the sensitivity drop in the instrument of $\sim 5.5 \times$ from a peak at 7100 to $10000 \AA$ and because moderately reddened $\mathrm{OB}$ stars are much fainter at longer wavelengths. For example, HD 27778 in Figure 1, with $E(B-V)=0.36$, drops by a factor of $\sim 2.5 \times$ over the same wavelength interval. Consequently, our G750L exposure times were limited to avoid saturation at the shortest wavelengths (assuming a mean extinction curve shape) and also to keep the total on-target time under 30 minutes to increase the potential for execution of our SNAP observations. As a result of these considerations, the $\mathrm{S} / \mathrm{Ns}$ of the G750L data vary considerably with wavelength and from star to star.

The G430L and G750L spectra are processed with the Instrument Definition Team (IDT) pipeline software written in IDL by D.J. Lindler in 1996-1997. This IDL data reduction is more flexible and offers the following advantages over the STScI pipeline results that are available from MAST ${ }^{6}$ : (1) better compensation for small shifts in pointing or instrumental flexure that may occur between the images of a cosmic-ray split (CRsplit) observation; (2) a larger extraction window height for the G750L data, to compensate for an increase in the spectral width in recent years; (3) elimination of "hot" pixels from the extracted spectrum; and (4) the use of tungsten lamp spectra to remove CCD fringing effects in the G750L spectra. The processing technique is discussed more thoroughly in Appendix A.

\footnotetext{
6 http://archive.stsci.edu/hst/
} 
Table 1

Basic Data for Program Stars

\begin{tabular}{|c|c|c|c|c|c|c|c|}
\hline$\overline{\text { Star }^{\mathrm{a}}}$ & $\begin{array}{c}\text { Spectral } \\
\text { Type }^{\mathrm{b}}\end{array}$ & $\begin{array}{c}V \\
(\mathrm{mag})\end{array}$ & $\begin{array}{c}E(B-V)^{\mathrm{c}} \\
(\mathrm{mag})\end{array}$ & $\begin{array}{l}\text { Distance }^{\mathrm{d}} \\
(\mathrm{pc})\end{array}$ & $\begin{array}{c}l \\
\left({ }^{\circ}\right)\end{array}$ & $\begin{array}{l}b \\
\left({ }^{\circ}\right)\end{array}$ & $\overline{\text { Reference }}$ \\
\hline $\mathrm{BD}+441080$ & B6 III & 9.12 & 0.85 & 521 & 162.484 & 1.51 & 1 \\
\hline BD+56 517 (NGC 869) & B $1.5 \mathrm{~V}$ & 10.50 & 0.56 & 2079 & 134.612 & -3.71 & 2 \\
\hline $\mathrm{BD}+56518(\mathrm{NGC} 869)$ & $\mathrm{B} 1.5 \mathrm{~V}$ & 10.60 & 0.59 & 2079 & 134.612 & -3.71 & 3 \\
\hline $\mathrm{BD}+56576(\mathrm{NGC} 884)$ & B2 III & 9.38 & 0.54 & 2345 & 135.047 & -3.59 & 4 \\
\hline $\mathrm{BD}+691231$ & B9.5 V & 9.27 & 0.23 & 389 & 110.255 & 11.36 & 5 \\
\hline $\mathrm{BD}+7192(\mathrm{Cr} 463)$ & $\ldots$ & 10.35 & 0.29 & 702 & 126.824 & 9.48 & $\ldots$ \\
\hline CPD-41 7715 (NGC 6231) & B2 IV-Vn & 10.53 & 0.44 & 1243 & 343.413 & 1.18 & 6 \\
\hline CPD-57 3507 (NGC 3293) & B1 V & 9.27 & 0.20 & 2910 & 285.832 & 0.08 & 7 \\
\hline CPD-57 3523 (NGC 3293) & B1 II & 8.02 & 0.27 & 2910 & 285.867 & 0.07 & 7 \\
\hline CPD-59 2591 (Tr 16) & $\ldots$ & 10.93 & 0.77 & 3200 & 287.591 & -0.74 & $\ldots$ \\
\hline CPD-59 $2600(\operatorname{Tr} 16)$ & O6 V((f)) & 8.61 & 0.51 & 3200 & 287.591 & -0.74 & 8 \\
\hline CPD-59 2625 (Tr 16) & $\mathrm{B} 2 \mathrm{~V}$ & 11.58 & 0.44 & 3200 & 287.637 & -0.68 & 9 \\
\hline GSC 03712-01870 & $\mathrm{O} 5+$ & 13.02 & 1.03 & 8200 & 137.859 & -0.97 & 10 \\
\hline HD 13338 & $\mathrm{~B} 1 \mathrm{~V}$ & 9.06 & 0.51 & 1506 & 133.513 & -3.27 & 11 \\
\hline HD 14250 (NGC 884) & B1 III & 8.97 & 0.58 & 2345 & 134.802 & -3.72 & 3 \\
\hline HD 14321 (NGC 884) & B1 IV & 9.22 & 0.53 & 2345 & 134.938 & -3.85 & 2 \\
\hline HD 17443 & B9 V & 8.74 & 0.38 & 284 & 133.924 & 7.55 & 12 \\
\hline HD 18352 & $\mathrm{~B} 1 \mathrm{~V}$ & 6.83 & 0.47 & 566 & 137.715 & 2.16 & 11 \\
\hline HD 27778 & B3 V & 6.36 & 0.36 & 262 & 172.759 & -17.38 & 13 \\
\hline HD 28475 & B5 V & 6.79 & 0.27 & 254 & 185.139 & -25.09 & 14 \\
\hline HD 29647 & B8 III & 8.31 & 1.00 & 157 & 174.042 & -13.35 & 15 \\
\hline HD 30122 & B5 III & 6.34 & 0.26 & 371 & 176.616 & -14.03 & 13 \\
\hline HD 30675 & B3 V & 7.53 & 0.52 & 355 & 173.597 & -10.20 & 16 \\
\hline HD 37061 (NGC 1978) & B $0.5 \mathrm{~V}$ & 6.83 & 0.53 & 2429 & 208.927 & -19.28 & 17 \\
\hline HD 38087 & $\mathrm{~B} 5 \mathrm{~V}$ & 8.30 & 0.30 & 315 & 207.073 & -16.26 & 17 \\
\hline HD 40893 & B0 IV: & 8.90 & 0.44 & 2632 & 180.091 & 4.33 & 11 \\
\hline HD 46106 (NGC 2244) & $\mathrm{B} 1 \mathrm{~V}$ & 7.92 & 0.41 & 1670 & 206.188 & -2.10 & 18 \\
\hline HD 46660 (NGC 2264) & $\mathrm{B} 1 \mathrm{~V}$ & 8.04 & 0.59 & 667 & 201.173 & 1.46 & 11 \\
\hline HD 54439 & B2 IIIn & 7.69 & 0.29 & 1295 & 225.402 & -1.69 & 11 \\
\hline HD 62542 & B5 V & 8.07 & 0.31 & 396 & 255.923 & -9.25 & 19 \\
\hline HD 68633 & B5 V & 8.00 & 0.48 & 274 & 266.224 & -9.55 & 20 \\
\hline HD 70614 & B6 & 9.27 & 0.67 & $\ldots$ & 259.807 & -3.24 & 20 \\
\hline HD 91983 (NGC 3293) & B1 III & 8.58 & 0.28 & 2910 & 285.882 & 0.05 & 21 \\
\hline HD 92044 (NGC 3293) & B0.5 II & 8.25 & 0.40 & 2910 & 285.937 & 0.06 & 21 \\
\hline HD $93028(\mathrm{Cr} 228)$ & O9 V & 8.37 & 0.21 & 2201 & 287.643 & -1.20 & 8 \\
\hline HD 93222 (Cr 228) & O7 III((f)) & 8.10 & 0.35 & 2201 & 287.726 & -1.01 & 8 \\
\hline HD 104705 & B0.5 III & 7.79 & 0.27 & 2082 & 297.465 & -0.34 & 11 \\
\hline HD 110336 & B9 IV & 8.64 & 0.45 & 333 & 302.463 & -14.50 & 22 \\
\hline HD 110946 & B1 V: & 9.14 & 0.50 & 1509 & 302.417 & -2.06 & 23 \\
\hline HD 112607 & B7/B8 III & 8.06 & 0.31 & 531 & 303.784 & -0.78 & 22 \\
\hline HD 142096 & $\mathrm{~B} 2.5 \mathrm{~V}$ & 5.03 & 0.15 & 94 & 350.724 & 25.38 & 24 \\
\hline HD 142165 & B6 IVn & 5.37 & 0.11 & 128 & 347.514 & 22.15 & 24 \\
\hline HD 146285 & B8 V & 7.93 & 0.32 & 208 & 351.012 & 18.20 & 24 \\
\hline HD 147196 & B5 V & 7.04 & 0.26 & 272 & 352.811 & 18.25 & 25 \\
\hline HD 147889 & $\mathrm{~B} 2 \mathrm{~V}$ & 7.90 & 1.09 & 153 & 352.868 & 17.04 & 26 \\
\hline HD 149452 & O8 $\mathrm{Vn}((\mathrm{f}))$ & 9.06 & 0.87 & 1636 & 337.455 & 0.04 & 8 \\
\hline HD 164073 & B3 III/IV & 8.03 & 0.20 & 961 & 344.171 & -12.56 & 20 \\
\hline HD 172140 & B0.5 III & 9.95 & 0.21 & 7318 & 5.274 & -10.61 & 27 \\
\hline HD 193322 & O9 V:((n)) & 5.83 & 0.38 & 727 & 78.110 & 2.78 & 8 \\
\hline HD 197512 & B1 V & 8.56 & 0.32 & 1614 & 87.895 & 4.63 & 16 \\
\hline HD 197702 & B1 III(n) & 7.89 & 0.46 & 701 & 73.925 & -6.83 & 28 \\
\hline HD 198781 & B $0.5 \mathrm{~V}$ & 6.45 & 0.32 & 768 & 99.956 & 12.62 & 11 \\
\hline HD 199216 & B1 II & 7.03 & 0.74 & 1097 & 88.922 & 3.04 & 29 \\
\hline HD $204827(\operatorname{Tr} 37)$ & $\mathrm{B} 0 \mathrm{~V}$ & 7.94 & 1.11 & 835 & 99.168 & 5.56 & 30 \\
\hline HD 210072 & B2 V & 7.66 & 0.49 & 659 & 100.844 & -0.37 & 26 \\
\hline HD 210121 & $\ldots$ & 7.67 & 0.33 & $\ldots$ & 56.861 & -44.47 & $\cdots$ \\
\hline HD 217086 (Сеp OB3) & O7 Vn & 7.64 & 0.92 & 724 & 110.215 & 2.73 & 8 \\
\hline HD 220057 & B2 IV & 6.93 & 0.23 & 770 & 112.116 & 0.20 & 31 \\
\hline HD $228969(\mathrm{Br} 86)$ & B2 II: & 9.49 & 0.99 & 2429 & 76.615 & 1.29 & 11 \\
\hline HD 236960 & B0.5 III & 9.75 & 0.72 & 3474 & 134.601 & -1.51 & 26 \\
\hline HD $239693(\operatorname{Tr} 37)$ & B3 V & 9.51 & 0.44 & 835 & 98.824 & 4.72 & 32 \\
\hline HD $239722(\operatorname{Tr} 37)$ & B2 IV & 9.55 & 0.92 & 835 & 100.339 & 5.05 & 32 \\
\hline HD $239745(\operatorname{Tr} 37)$ & $\mathrm{B} 1 \mathrm{~V}$ & 8.91 & 0.53 & 835 & 99.432 & 2.99 & 32 \\
\hline
\end{tabular}


Table 1

(Continued)

\begin{tabular}{lccccrr}
\hline \hline Star $^{\mathrm{a}}$ & $\begin{array}{c}\text { Spectral } \\
\text { Type }^{\mathrm{b}}\end{array}$ & $\begin{array}{c}V \\
(\mathrm{mag})\end{array}$ & $\begin{array}{c}E(B-V)^{\mathrm{c}} \\
(\mathrm{mag})\end{array}$ & $\begin{array}{c}\text { Distance } \\
(\mathrm{pc})\end{array}$ & $\begin{array}{c}l \\
\left({ }^{\mathrm{d}}\right)\end{array}$ & $\begin{array}{c}b \\
\left({ }^{\circ}\right)\end{array}$ \\
\hline HD 282485 & B9 V & 9.88 & 0.47 & 412 & 172.220 & -10.29 \\
HD 292167 & O9 III: & 9.25 & 0.69 & 2681 & 211.630 & -1.17 \\
HD 294264 (NGC 1977) & B3 Vn & 9.53 & 0.49 & 476 & 208.492 & -19.16 \\
HD 303068 (NGC 3293) & B1 V & 9.78 & 0.25 & 2910 & 285.688 & 0.07 \\
NGC 2244 11 (NGC 2244) & B2 V & 9.73 & 0.44 & 1670 & 206.422 & -2.02 \\
NGC 2244 23 (NGC 2244) & B2.5 V & 11.21 & 0.47 & 1670 & 206.311 & -2.08 \\
Trumpler 14 6 (Tr 14) & B1 V & 11.23 & 0.49 & 3200 & 287.419 & -0.59 \\
Trumpler 14 27 (Tr 14) & $\ldots$ & 11.30 & 0.57 & 3200 & 287.399 & -0.60 \\
VSS VIII-10 & B8 V & 10.07 & 0.73 & 235 & 359.518 & -18.29 \\
\hline
\end{tabular}

Notes.

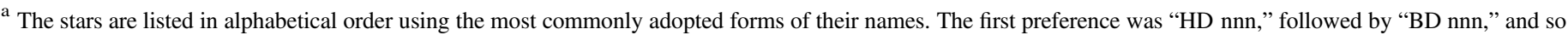

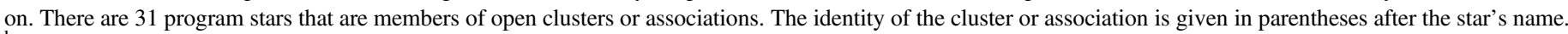

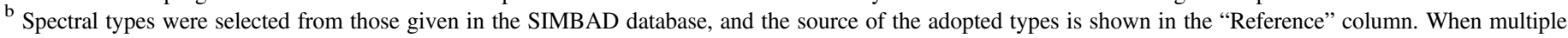
types were available for a particular star, we selected one based on our own preferred ranking of the sources.

${ }^{c}$ Values of $E(B-V)$ were inferred from the values of $E(44-55)$ determined from the extinction curves, whose construction is described in Section 3.2 .

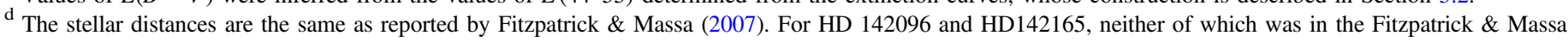

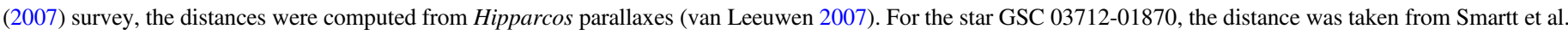
(1996).

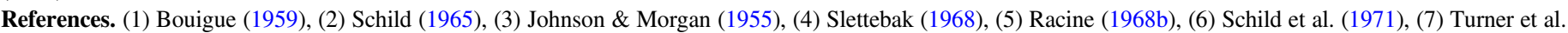

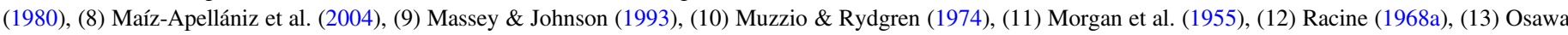

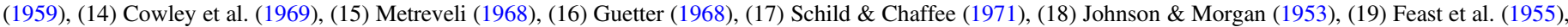

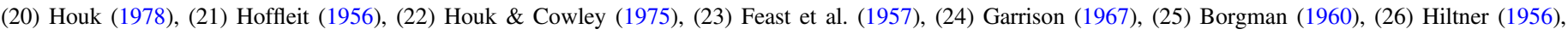

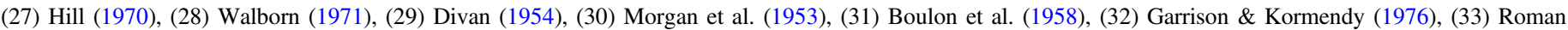
(1955), (34) Smith (1972), (35) Meadows (1961), (36) Johnson (1962), (37) Morrell et al. (1988), (38) Vrba \& Rydgren (1984).

In addition to the above data extraction advantages, our postprocessing includes the charge transfer efficiency (CTE) corrections of Goudfrooij et al. (2006) and an up-to-date correction for the changes in STIS sensitivity with time and temperature. The observations of the three primary flux standards G191B2B, GD153, and GD71, as corrected for CTE and the latest measures of sensitivity change with time, produce the current absolute flux calibration (CALSPEC; see Bohlin et al. 2014).

After completion of all the processing steps, the data were concatenated, with G430L used below $5450 \AA$ and G750L used above $5450 \AA$ A.

\subsection{Ultraviolet Data: IUE}

With the exception of GSC 03712-01870, the stars included in this paper have all been incorporated in previous UV extinction studies, notably by Fitzpatrick \& Massa (2005a, hereafter F05) and F07, and have IUE spectrophotometric observations available from both the short-wavelength region ("SWP" camera; 1150-1980 ^) and long-wavelength region ("LWR" or "LWP" cameras; 1980-3200 A). Only SWP observations exist for GSC 03712-01870. The IUE data were obtained by us from the MAST archive and were processed as described earlier (e.g., see F07). The critical step in the processing of the archival data was the correction for deficiencies in the IUE NEWSIPS calibration, using the results of Massa \& Fitzpatrick (2000, hereafter M00). These deficiencies include systematic thermal and temporal effects and an incorrect absolute calibration. This is particularly important for our current study because we derive extinction curves using model atmosphere calculations, rather than unreddened comparison stars, and errors in the calibration of the data would be reflected directly in the resultant curves.
The M00 corrections place the IUE data on the absolute flux calibration system as it was in the year 2000. The calibration has been modified in the intervening years, and to take advantage of this, we additionally corrected the data to the current CALSPEC standard. We did this by comparing M00-corrected IUE spectra of the flux standards G191B2B, GD71, and GD153 with the current SEDs of those stars as found in the CALSPEC database. ${ }^{7}$ This comparison indicated that the flux levels of M00-corrected IUE spectra need to be reduced by an average of $\sim 0.9 \%$ in the short-wavelength region and $\sim 1.8 \%$ in the long-wavelength region (with a roughly linear wavelength dependence across the whole $I U E \mathrm{UV})$. This final correction makes the calibration of the IUE data fully consistent and compatible with the calibration of the optical HST spectrophotometry described above and the NIR photometry described below. When the processing was completed, the UV spectra for each star were trimmed to the wavelength range 1150-3000 $\AA$, with the shortand long-wavelength camera data joined at a wavelength of $1978 \AA$ A.

\subsection{NIR Data: 2MASS}

The third data set consists of NIR $J H K$ photometry from 2MASS. These data were obtained from the 2MASS All-Sky Point Source Catalog, accessed via the 2MASS website hosted by the Infrared Processing and Analysis Center. ${ }^{8} \mathrm{JHK}$ measurements are available for all program stars, although, because of the lack of uncertainty estimates, we excluded the $J$ and $H$ data

\footnotetext{
7 The CALSPEC Calibration Database was accessed at http://www.stsci.edu/ hst/instrumentation/reference-data-for-calibration-and-tools/astronomical-catalogs/ calspec and data retrieved from $\mathrm{ftp}: / / \mathrm{ftp}$.stsci.edu/cdbs/calspec/.

8 The 2MASS website can be found at https://www.ipac.caltech.edu/ 2 mass/.
} 


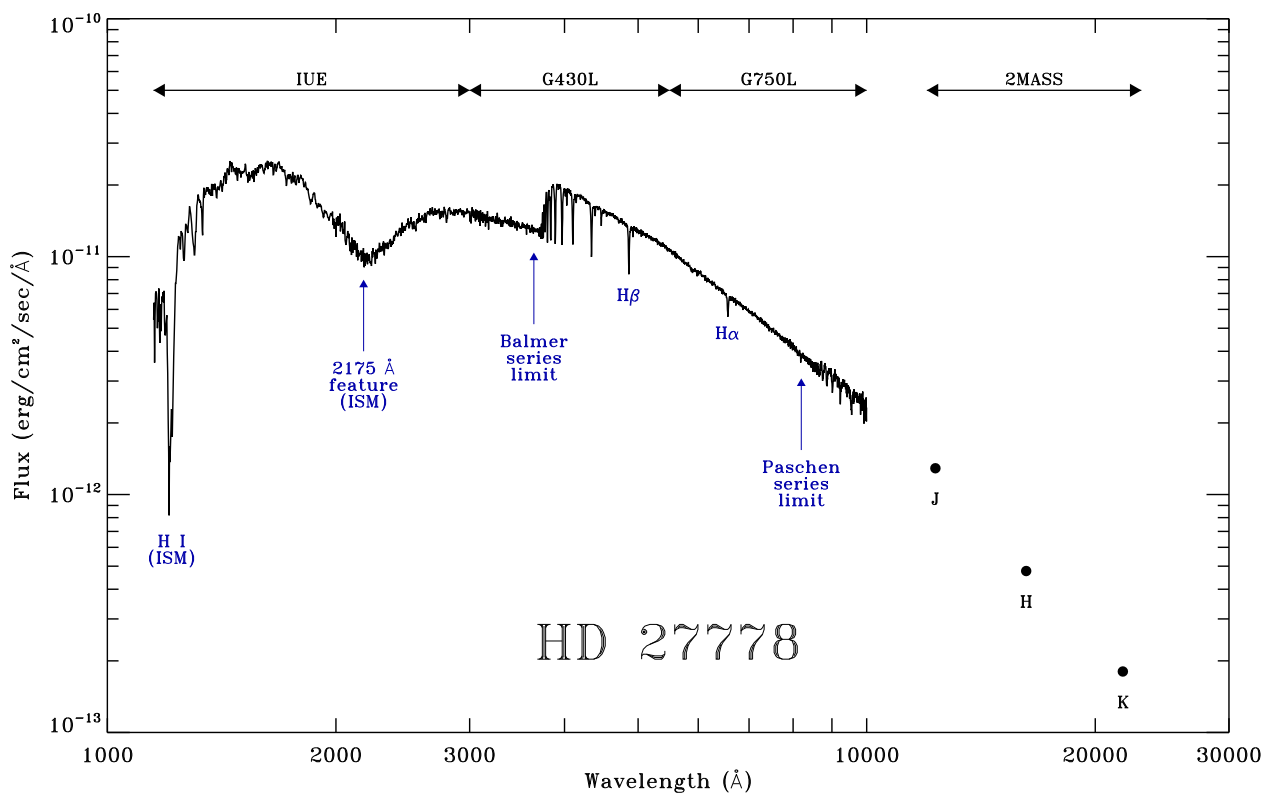

Figure 1. Representative SED for our program stars, using HD 27778 as an example. Sources and wavelength ranges of the various data sets are indicated at the top of the figure. International Ultraviolet Explorer satellite data from the SWP, LWP, and LWR cameras, covering the range 1150-3000 $\AA$, were obtained from the Mikulski Archive for Space Telescopes. New Hubble Space Telescope spectrophotometric observations using the G430L and G750L gratings were acquired for this program and cover the ranges 3000-5450 $\AA$ and 5450-10000 $\AA$, respectively. NIR $J H K$ photometry from 2MASS was obtained from the 2MASS archive maintained by the Infrared Processing and Analysis Center. Prominent stellar and interstellar features are identified beneath the SED.

for the star CPD-57 3523. The 2MASS data required no additional processing.

The flux calibration of the 2MASS bands is important to our study because we derive NIR extinction by comparing the 2MASS magnitudes for reddened stars with synthetic values derived from the stars' assumed intrinsic SEDs. Thus, the synthetic photometry must be calibrated in a manner consistent with the latest CALSPEC system. The standard 2MASS calibration was performed by Cohen et al. (2003), based on a synthetic spectrum of Vega (see Cohen et al. 1992) and the assumption that Vega defines zero magnitude in the 2MASS NIR. To ensure consistency with our new study, we rederived this calibration, based on the latest CALSPEC database. We did this by computing synthetic 2MASS magnitudes for a subset of the CALSPEC calibration sample and then determining the transformation that maps these values to the observed 2MASS $J H K$ magnitudes. The procedure is essentially identical to that used by Fitzpatrick \& Massa (2005b) in a general calibration of synthetic photometry for B and early A-type stars. In this case, we began with the 40 flux standard stars from the CALSPEC Calibration Database for which NIR model fluxes were computed. This subset of the full calibration sample includes all of the stars in Table 1 of the database for which entries exist in Column (6), indicating the existence of a model SED, with the exception of SF1615+001A (no 2MASS data) and HZ43 (contamination of 2MASS by a red companion star). The sample was further restricted to those stars with 2MASS magnitude uncertainties less than $0.1 \mathrm{mag}$, leaving 37,36 , and 32 stars for calibration of the $J$, $H$, and $K$ filters, respectively. We performed synthetic photometry on the model SEDs (in $f_{\lambda}$ units) using the 2MASS $J H K$ filter response functions and $F_{\lambda}(0 \mathrm{mag})$ values from Cohen et al. (2003). The transformation to the observed 2MASS system is expressed in terms of "zero-point offsets" as defined in Cohen et al. (2003) or, equivalently, in Equation (1) of Maíz Apellániz (2007). The results for each filter are shown in Figure 2, where we plot zero-point offsets against the 2MASS magnitudes. The final adopted offsets are weighted means with values of $-0.023,+0.002$, and
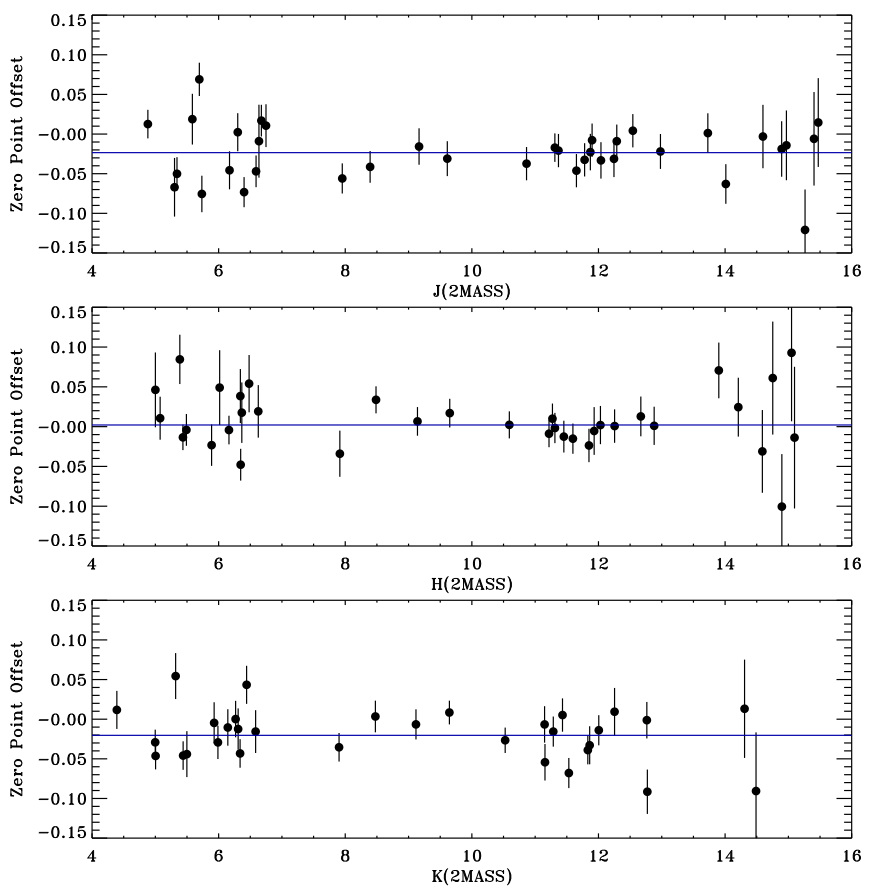

Figure 2. Calibration of synthetic 2MASS JHK photometry. Synthetic $J H K$ magnitudes were computed for the model SEDs of 30-40 stars in the CALSPEC database, as described in Section 2.4, and "zero-point offsets" determined to match the synthetic values to the observed 2MASS values. The top, middle, and bottom panels show these offsets for the $J, H$, and $K$ filters, respectively, plotted against the observed 2MASS magnitudes. Thick blue lines indicate the weighted-mean offsets, with values of $-0.023,0.002$, and -0.020 mag for $J, H$, and $K$, respectively. The error bars are those associated with the observed magnitudes.

-0.020 mag for the $J, H$, and $K$ filters, respectively. These offsets are almost identical (i.e., within $\pm 0.005 \mathrm{mag}$ ) to those found by Maíz Apellániz \& Pantaleoni González (2018), who also used the 
CALSPEC database to examine the 2MASS calibration. Like Maíz Apellániz \& Pantaleoni González (2018), we find no evidence for color dependence in these offsets, nor for a systematic brightness dependence (as can be seen in Figure 2). Use of these zero-point offsets ensures that the 2MASS photometric data are calibrated consistently with our UV and optical spectrophotometric data sets.

\section{Creating the NIR-UV Extinction Curves}

We derive NIR-through-UV extinction curves for our 72 program stars using the "Extinction without Standards" approach, first described by F05. This technique utilizes SEDs from stellar atmosphere models to represent the intrinsic SEDs of the reddened program stars, rather than observations of unreddened stars of similar spectral type. The advantages of such an approach are described in detail in F05. In our previous applications of Extinction without Standards (e.g., F05 and F07), we determined the stellar properties and the extinction properties simultaneously by fitting IUE UV spectrophotometry and optical/near-IR photometry with model SEDs and a parameterized version of the interstellar extinction curve. In this study, we adopt a slightly different approach, explicitly separating the process into two distinct steps: (1) first, we determine the appropriate stellar properties needed to characterize the model SED, and then (2) form a normalized ratio between the observed and model SEDs (i.e., the extinction curve). This approach is made possible by the new $H S T$ spectrophotometry, which allows a detailed modeling of critical stellar diagnostics in the optical spectral region. The advantage is that the resultant extinction curves are derived completely empirically, without any assumptions as to their underlying functional form. The two steps in this process are described separately in the following subsections.

\subsection{Step 1: Determining the Stellar Properties}

We estimate the intrinsic properties of our program stars by fitting their G430L spectra with stellar atmosphere models. The HST G430L data consist of spectrophotometric observations over the wavelength range $2900-5700 \AA$ at a resolution better than $10 \AA$. As such, they provide a clear view of the wide range of spectroscopic features present in the classical blue spectral region, which formed the basis of the spectral classification system. Thus, ample information is present in these data to determine stellar effective temperature $\left(T_{\text {eff }}\right)$, surface gravity $(\log g)$, and, more coarsely, metallicity $([\mathrm{m} / \mathrm{H}])$.

For most of our program stars, we fit the G430L data using the TLUSTY NLTE SED grids published by Lanz \& Hubeny (2003, "OSTAR2002") and Lanz \& Hubeny (2007, "BSTAR2006"). The grids were computed at full spectral resolution (at the thermal broadening level) and, together, cover a wide range of metallicities $([\mathrm{m} / \mathrm{H}]=+0.3$ to -1.0$)$, effective temperatures $\left(T_{\text {eff }}=15,000 \mathrm{~K}\right.$ to $55,000 \mathrm{~K}$ ), and surface gravities ( $\log g=4.75$ to the modified Eddington limit). The OSTAR2002 grid assumes a microturbulence velocity of $v_{\text {turb }}=10 \mathrm{~km} \mathrm{~s}^{-1}$, while the BSTAR2006 grid assumes $v_{\text {turb }}=2 \mathrm{~km} \mathrm{~s}^{-1}$, with a small number of low-gravity models computed with $v_{\text {turb }}=10 \mathrm{~km} \mathrm{~s}^{-1}$. Both grids assume the solar abundance scale of Grevesse \& Sauval (1998). The modeling process involves four steps: (1) begin with initial estimates of $T_{\text {eff }}$, $\log g$, and $[\mathrm{m} / \mathrm{H}] ;$ (2) interpolate within the appropriate TLUSTY grid to produce a full-resolution $\mathrm{SED}, \mathrm{F}_{\lambda}$, with these properties in the G430L spectral range; (3) broaden the SED to account for the effects of stellar rotation and the instrumental profile of the G430L; and (4) compare the model SED with the G430L spectrum, using $\chi^{2}$ as the measure of the goodness of fit. The initial estimates of the stellar properties were then adjusted and the process repeated iteratively until the minimum value of $\chi^{2}$ was achieved. The broadband shapes and levels of the G430L SEDs are distorted relative to the models because of the effects of distance and interstellar extinction. To remove these effects from the analysis, we mapped the overall shapes of the model continua onto the observed continua using a cubic spline, whose anchor points were iteratively adjusted along with the stellar properties. Thus, the final stellar properties determined from the fitting procedure depend only on the absorption line spectrum (plus the Balmer jump) and not at all on the general shape of the spectrum. The continuum mapping is not used in the subsequent derivation of the extinction curves.

The broadening applied to the model SEDs requires some additional discussion. In early tests, we applied a Gaussian point-spread function (PSF) with FWHM $=5.5 \AA$, as taken from the STIS Instrument Handbook (Riley 2019), ${ }^{9}$ and rotational broadening based on $v \sin i$ values from the literature. In a number of cases, however, the observed spectra appeared significantly more broadened, consistent (in the most extreme cases) with a PSF FWHM of $7.8 \AA$. The initially adopted value $5.5 \AA$ appeared, in these tests, to be a minimum. We attribute these discrepancies to a variable PSF width in our slitless spectra, arising from focus changes due to thermal cycling of the instrument (also known as "breathing"). Ideally, we would deal with this by adopting measured $v \sin i$ values for all of the stars and allowing the FWHM of the PSF to be a free parameter in the fitting routine, adjusted to achieve a best fit to the line profiles. Unfortunately, only about one-third of our program stars have measured $v$ sin $i$ values, so this is not feasible. Instead, we adopted a modified approach and fixed the PSF FWHM at the minimum observed value of $5.5 \AA$ and varied the width of the rotational broadening profile to achieve a best fit to the spectra. This yields a value of " $v_{\text {broad }}$ " which contains both the rotational broadening of the star and the effects of breathing. We found that, in cases where $v \sin i$ is known, these two different approaches yield essentially identical results, in both the values of $\chi^{2}$ and the model parameters. Evidently, the resolution and $\mathrm{S} / \mathrm{N}$ of our data are such that the differences between a Gaussian and a rotational profile are not significant.

Eleven of the program stars have $T_{\text {eff }}$ values below the $15,000 \mathrm{~K}$ lower limit of the TLUSTY BSTAR2006 grid. The general procedure for determining their properties is similar to that described above, except that in step (2) we used a combination of the ATLAS9 LTE models from Kurucz (1991) to produce an interpolated atmospheric structure for the desired stellar properties, and then the spectral synthesis program SPECTRUM (Version 2.76e) developed by Richard Gray (see Gray \& Corbally 1994) to produce a fully resolved stellar spectrum over the G430L spectral window. For consistency with the TLUSTY grids, these calculations also assumed the Grevesse \& Sauval (1998) solar abundance scale.

The ability of the stellar models to reproduce the G430L spectra is demonstrated in Figure 3. The various panels show the observed G430L data (small black filled circles) overlaid with the best-fit models (solid blue lines). For clarity, the stars are ordered by decreasing $T_{\text {eff }}$, from the top of Figure 3(a) (hottest star) to the bottom of Figure 3(g) (coolest star). Prominent stellar features are identified and labeled in green,

\footnotetext{
9 The STIS Instrument Handbook was accessed at http://www.stsci.edu/hst/ stis/faqs/documents/handbooks/currentIHB/cover.html.
} 


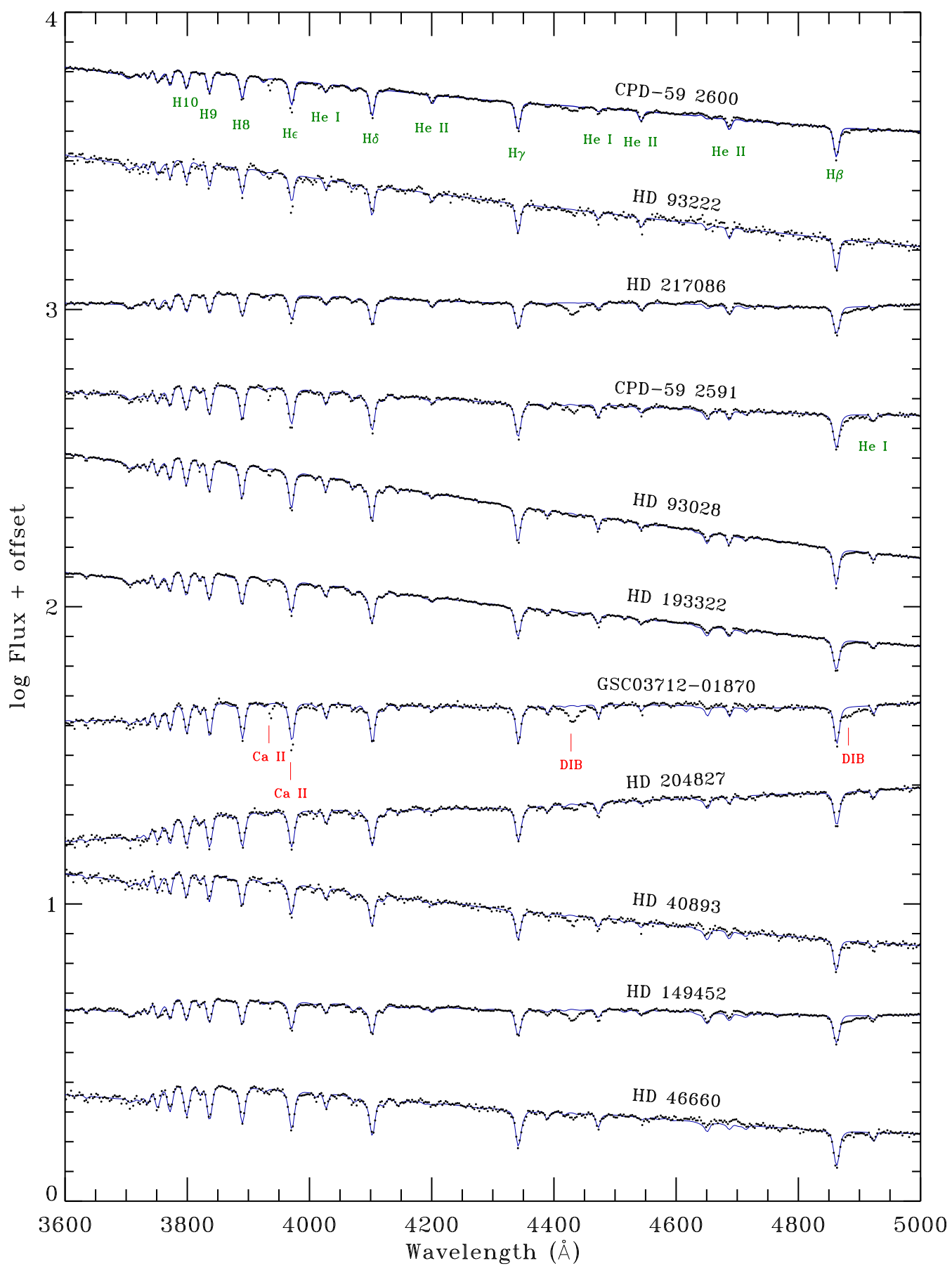

Figure 3. Model atmosphere fits to the G430L spectra of the program stars. Small black circles show the observed G430L spectrum over the wavelength range 3600-5000 A. Solid blue curves show the best-fitting stellar atmosphere models. The fitting procedure is described in Section 3.1 and included the full range of the G430L spectra (2900-5700 ̊). Most of the spectral features are contained in the narrower range shown in the figure. Prominent stellar features are identified in green, and interstellar features, including gas-phase absorption lines and diffuse interstellar bands, are identified in red. The stars are ordered in decreasing $T_{\mathrm{eff}}$ throughout the figures. Stellar properties derived from the fits are listed in Table 2.

(The complete figure set (seven images) is available.)

while interstellar features are labeled in red. As seen in the figures, the models reproduce the observed stellar features very well. The only significant and systematic discrepancies noticeable in Figure 3 are due to interstellar features, notably the broad diffuse interstellar bands (DIBs) at $4430 \AA$ and $4870 \AA$ and the Ca II H and K absorption lines at $3968 \AA$ and $3933 \AA$, respectively, which are identified in the first panel of Figure 3. Four stars in the sample (HD 147196, HD 197702, HD 292167 , and VSS VII-10) show evidence for mild $\mathrm{H} \alpha$ emission in their G750L spectra, which is not reproduced by the atmosphere models. Two of these stars (HD 197702 and HD 292167) are early-type giants at the low-gravity edge of our adopted model grids $(\log g \sim 3.0)$. The other two are late-B main-sequence stars and may be rapid rotators. Indeed, HD 147196 has the

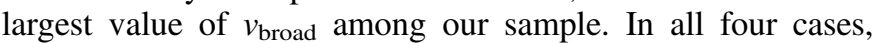
however, the blue region of the spectra appears normal (as seen 
Table 2

Stellar Properties from G430L Analysis

\begin{tabular}{|c|c|c|c|c|c|c|}
\hline Star & $\begin{array}{l}T_{\text {eff }} \\
(\mathrm{K})\end{array}$ & $\log g$ & {$[\mathrm{~m} / \mathrm{H}]^{\mathrm{a}}$} & $\begin{array}{c}v_{\text {turb }} \mathrm{b}^{\mathrm{b}} \\
\left(\mathrm{km} \mathrm{s}^{-1}\right)\end{array}$ & $\begin{array}{c}v_{\text {broad }}^{\mathrm{c}} \\
\left(\mathrm{km} \mathrm{s}^{-1}\right)\end{array}$ & $\begin{array}{c}\text { Atmosphere } \\
\text { Model }^{\mathrm{d}}\end{array}$ \\
\hline $\mathrm{BD}+441080$ & 12879 & 3.42 & -0.25 & 2 & 303 & ATLAS9/SPECTRUM \\
\hline $\mathrm{BD}+56517$ & 23752 & 3.76 & -0.38 & 2 & 192 & TLUSTY B02 \\
\hline $\mathrm{BD}+56518$ & 23415 & 3.80 & -0.36 & 2 & 165 & TLUSTY B02 \\
\hline $\mathrm{BD}+56576$ & 23275 & 3.48 & -0.16 & 2 & 169 & TLUSTY B02 \\
\hline $\mathrm{BD}+691231$ & 11292 & 4.23 & -0.16 & 2 & 413 & ATLAS9/SPECTRUM \\
\hline $\mathrm{BD}+7192$ & 11634 & 4.04 & -0.21 & 2 & 325 & ATLAS9/SPECTRUM \\
\hline CPD-41 7715 & 23617 & 3.98 & -0.23 & 2 & 322 & TLUSTY B02 \\
\hline CPD-57 3507 & 25921 & 3.71 & -0.19 & 2 & 192 & TLUSTY B02 \\
\hline CPD-57 3523 & 25037 & 3.10 & -0.34 & 10 & 255 & TLUSTY B10 \\
\hline CPD-59 2591 & 34169 & 4.18 & -0.25 & 10 & 332 & TLUSTY O10 \\
\hline CPD-59 2600 & 38901 & 3.68 & -0.22 & 10 & 338 & TLUSTY O10 \\
\hline CPD-59 2625 & 26310 & 4.42 & -1.03 & 2 & 137 & TLUSTY B02 \\
\hline GSC 03712-01870 & 33233 & 3.98 & -0.50 & 10 & 229 & TLUSTY O10 \\
\hline HD 13338 & 22748 & 3.61 & -0.38 & 2 & 204 & TLUSTY B02 \\
\hline HD 14250 & 24701 & 3.53 & -0.16 & 2 & 350 & TLUSTY B02 \\
\hline HD 14321 & 23294 & 3.57 & -0.33 & 2 & 224 & TLUSTY B02 \\
\hline HD 17443 & 11241 & 4.15 & -0.16 & 2 & 397 & ATLAS9/SPECTRUM \\
\hline HD 27778 & 17362 & 3.91 & -0.47 & 2 & 295 & TLUSTY B02 \\
\hline HD 28475 & 15570 & 4.01 & -0.44 & 2 & 368 & TLUSTY B02 \\
\hline HD 29647 & 12356 & 3.58 & -0.25 & 2 & 286 & ATLAS9/SPECTRUM \\
\hline HD 30122 & 16432 & 3.65 & -1.05 & 2 & 341 & TLUSTY B02 \\
\hline HD 30675 & 16428 & 3.97 & -0.37 & 2 & 318 & TLUSTY B02 \\
\hline HD 37061 & 28366 & 4.12 & -0.51 & 10 & 332 & TLUSTY O10 \\
\hline HD 38087 & 17408 & 4.20 & -0.34 & 2 & 294 & TLUSTY B02 \\
\hline HD 40893 & 31837 & 3.60 & -0.19 & 10 & 335 & TLUSTY O10 \\
\hline HD 46106 & 29265 & 4.10 & -0.58 & 10 & 339 & TLUSTY O10 \\
\hline HD 46660 & 31067 & 3.98 & -0.53 & 10 & 303 & TLUSTY O10 \\
\hline HD 54439 & 25300 & 3.71 & -0.27 & 2 & 315 & TLUSTY B02 \\
\hline HD 62542 & 17083 & 4.07 & -0.55 & 2 & 302 & TLUSTY B02 \\
\hline HD 68633 & 18643 & 3.91 & -0.52 & 2 & 277 & TLUSTY B02 \\
\hline HD 70614 & 18105 & 3.25 & -0.39 & 2 & 333 & TLUSTY B02 \\
\hline HD 91983 & 24490 & 3.36 & -0.12 & 2 & 232 & TLUSTY B02 \\
\hline HD 92044 & 24529 & 3.06 & -0.28 & 10 & 247 & TLUSTY B10 \\
\hline HD 93028 & 33564 & 4.04 & -0.21 & 10 & 252 & TLUSTY O10 \\
\hline HD 93222 & 36349 & 3.66 & -0.24 & 10 & 296 & TLUSTY O10 \\
\hline HD 104705 & 29222 & 3.44 & -0.02 & 10 & 307 & TLUSTY O10 \\
\hline HD 110336 & 11941 & 4.15 & -0.17 & 2 & 300 & ATLAS9/SPECTRUM \\
\hline HD 110946 & 20582 & 3.24 & -0.53 & 10 & 249 & TLUSTY B10 \\
\hline HD 112607 & 12980 & 3.43 & -0.22 & 2 & 347 & ATLAS9/SPECTRUM \\
\hline HD 142096 & 18205 & 4.34 & -0.29 & 2 & 330 & TLUSTY B02 \\
\hline HD 142165 & 14230 & 4.20 & -0.31 & 2 & 398 & ATLAS9/SPECTRUM \\
\hline HD 146285 & 12328 & 4.36 & -0.18 & 2 & 353 & ATLAS9/SPECTRUM \\
\hline HD 147196 & 12225 & 3.94 & -0.45 & 2 & 544 & ATLAS9/SPECTRUM \\
\hline HD 147889 & 22061 & 4.13 & -0.54 & 2 & 229 & TLUSTY B02 \\
\hline HD 149452 & 31816 & 3.55 & -0.00 & 10 & 405 & TLUSTY O10 \\
\hline HD 164073 & 17573 & 3.99 & -0.43 & 2 & 333 & TLUSTY B02 \\
\hline HD 172140 & 27347 & 3.77 & 0.06 & 10 & 232 & TLUSTY O10 \\
\hline HD 193322 & 33358 & 3.90 & -0.19 & 10 & 387 & TLUSTY O10 \\
\hline HD 197512 & 23773 & 3.83 & -0.41 & 2 & 165 & TLUSTY B02 \\
\hline HD 197702 & 23854 & 2.99 & -0.38 & 10 & 430 & TLUSTY B10 \\
\hline HD 198781 & 25529 & 3.13 & -0.49 & 10 & 284 & TLUSTY B10 \\
\hline HD 199216 & 24140 & 3.04 & -0.34 & 10 & 189 & TLUSTY B10 \\
\hline HD 204827 & 32037 & 3.96 & -0.28 & 10 & 345 & TLUSTY O10 \\
\hline HD 210072 & 19186 & 3.08 & -0.55 & 10 & 312 & TLUSTY B10 \\
\hline HD 210121 & 16498 & 4.06 & -0.29 & 2 & 290 & TLUSTY B02 \\
\hline HD 217086 & 35722 & 3.65 & -0.34 & 10 & 443 & TLUSTY O10 \\
\hline HD 220057 & 19736 & 4.21 & -0.38 & 2 & 396 & TLUSTY B02 \\
\hline HD 228969 & 28818 & 3.84 & -0.28 & 10 & 378 & TLUSTY O10 \\
\hline HD 236960 & 26572 & 3.47 & 0.11 & 2 & 229 & TLUSTY B02 \\
\hline HD 239693 & 18885 & 4.11 & -0.33 & 2 & 357 & TLUSTY B02 \\
\hline HD 239722 & 20146 & 3.79 & -0.62 & 2 & 210 & TLUSTY B02 \\
\hline HD 239745 & 27059 & 4.12 & -0.37 & 2 & 215 & TLUSTY B02 \\
\hline HD 282485 & 12927 & 3.88 & -0.26 & 2 & 309 & ATLAS9/SPECTRUM \\
\hline
\end{tabular}


Table 2

(Continued)

\begin{tabular}{|c|c|c|c|c|c|c|}
\hline Star & $\begin{array}{l}T_{\text {eff }} \\
(\mathrm{K})\end{array}$ & $\log g$ & {$[\mathrm{~m} / \mathrm{H}]^{\mathrm{a}}$} & $\begin{array}{c}v_{\text {turb }}{ }^{\mathrm{b}} \\
\left(\mathrm{km} \mathrm{s}^{-1}\right)\end{array}$ & $\begin{array}{c}v_{\text {broad }}{ }^{\mathrm{c}} \\
\left(\mathrm{km} \mathrm{s}^{-1}\right)\end{array}$ & $\begin{array}{l}\text { Atmosphere } \\
\text { Model }^{\mathrm{d}}\end{array}$ \\
\hline HD 292167 & 28817 & 3.01 & -0.21 & 10 & 225 & TLUSTY O10 \\
\hline HD 294264 & 19095 & 4.23 & -0.37 & 2 & 300 & TLUSTY B02 \\
\hline HD 303068 & 24810 & 3.80 & -0.29 & 2 & 203 & TLUSTY B02 \\
\hline NGC 224411 & 27166 & 4.13 & -0.37 & 2 & 296 & TLUSTY B02 \\
\hline NGC 224423 & 19091 & 4.06 & -0.29 & 2 & 440 & TLUSTY B02 \\
\hline Trumpler 146 & 28047 & 4.09 & -0.22 & 2 & 243 & TLUSTY B02 \\
\hline Trumpler 1427 & 25998 & 4.17 & -0.45 & 2 & 178 & TLUSTY B02 \\
\hline VSS VIII-10 & 11525 & 3.76 & -0.30 & 2 & 393 & ATLAS9/SPECTRUM \\
\hline
\end{tabular}

Notes.

a Values of $[\mathrm{m} / \mathrm{H}]$ are measured with respect to the solar abundance scale of Grevesse \& Sauval (1998).

${ }^{\mathrm{b}}$ Values of the microturbulence velocity $v_{\text {turb }}$, either $2 \mathrm{~km} \mathrm{~s}^{-1}$ or $10 \mathrm{~km} \mathrm{~s}^{-1}$, were determined by the grid of model atmospheres used to model each star.

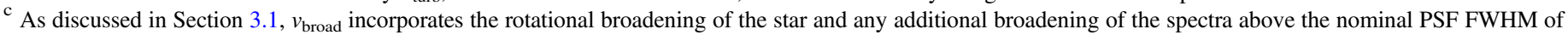
$5.5 \AA$ A, due to "breathing" of the instrument.

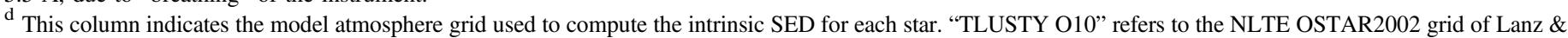

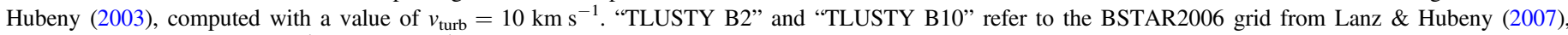

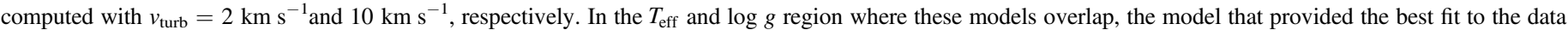

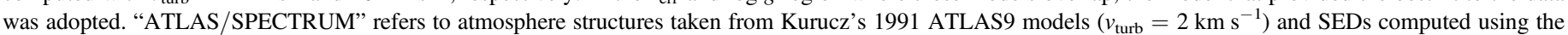
spectral synthesis program SPECTRUM from Gray \& Corbally (1994). These models were used for the coolest stars in the sample, with $T_{\text {eff }} \leqslant 15,000$ K.

in Figure 3), as does the UV region, and we believe that the stellar parameters and SEDs are well reproduced by the models used here. (Note that the rapidly rotating HD 147196 will ultimately be excluded from the final extinction sample analyzed below due to its low value of $E(B-V)$.)

The final sets of parameters describing the best-fit models are listed in Table 2. In addition to the values of $T_{\text {eff }}, \log g[\mathrm{~m} / \mathrm{H}]$, $v_{\text {turb }}$, and $v_{\text {broad }}$, we also indicate-in the last column of the table - the particular stellar atmosphere grid used for each star. In cases where the stellar properties fell into regions in which the different TLUSTY grids overlapped, we chose the model that yielded the best fit to the data.

The typical statistical uncertainties in the stellar parameters derived from the fitting procedure are $\pm 200 \mathrm{~K}$ in $T_{\text {eff }}, \pm 0.02$ in $\log g, \pm 0.1$ in $[\mathrm{m} / \mathrm{H}]$, and $\pm 10 \mathrm{~km} \mathrm{~s}^{-1}$ in $v_{\text {broad. }}$ These, however, do not take into account systematic effects that could arise from the fundamental assumptions in the modeling process. For example, the solar abundances of Grevesse \& Sauval (1998) have been superceded since the TLUSTY grids were computed (Grevesse et al. 2013). In addition, the overlap region between the cool TLUSTY BSTAR2006 models and the ATLAS9/ SPECTRUM models is illustrative. For any star for which the BSTAR2006 models indicated $T_{\text {eff }}<15,000 \mathrm{~K}$, we adopted the ATLAS9/SPECTRUM combination described above. However, when experimenting with the ATLAS9/SPECTRUM combination for stars warmer than $15,000 \mathrm{~K}$, we found that they yielded $T_{\text {eff }}$ values several hundred $\mathrm{K}$ hotter than the best-fit TLUSTY models. This is undoubtedly due to the fundamental difference in the LTE versus NLTE approaches and, possibly, to differences in the base opacities and atomic parameters assumed in the models. It would be useful to repeat this comparison with other LTE model grids, such as the BOSZ grid of Bohlin et al. (2017). However, while this is an interesting stellar atmospheres issue, fortunately for us it has little impact on the derived extinction curves. As was shown by Massa et al. (1983), the effects of temperature mismatch in extinction curves formed from early-type stars tend to be self-canceling due to the optical normalization (which, with some modifications, we will adopt here). Moreover, the wide range in intrinsic extinction curve properties also serves to lessen the impact of the relatively small uncertainties on the intrinsic stellar properties.

\subsection{Step 2: Constructing the Extinction Curves}

Once the properties of the reddened stars were determined, the production of the normalized extinction curves was straightforward. Here, $A(\lambda)$, the total extinction at a wavelength $\lambda$, is given by

$$
A(\lambda)=-2.5 \times \log \frac{f_{\lambda}}{F_{\lambda}}+5 \times \log \frac{R_{*}}{d},
$$

where $f_{\lambda}$ and $F_{\lambda}$ are the observed and intrinsic SEDs, respectively, and the quantity $R_{*} / d$ is the angular radius of the star, that is, its physical radius $R_{*}$ divided by its distance $d$. Because these latter two quantities are rarely known accurately, extinction is usually presented in a normalized form, in which the angular radius cancels out. In this paper, we adopt the normalization

$$
k(\lambda-55) \equiv \frac{E(\lambda-55)}{E(44-55)}=\frac{A(\lambda)-A(55)}{A(44)-A(55)}
$$

and

$$
R(55) \equiv \frac{A(55)}{E(44-55)} .
$$

These are analogous to the most commonly used normalizations of extinction, $k(\lambda-V) \equiv E(\lambda-V) / E(B-V)$ and $R(V)$, but with monochromatic measures of the extinction at 4400 and $5500 \AA$ substituting for measurements with the Johnson $B$ and $V$ filters, respectively. The monochromatic values were determined by interpolation of a quadratic fit to the extinction curves over a range $\pm 0.1 \mu \mathrm{m}^{-1}$ from the desired wavelength. The benefit of the monochromatic normalization is that it eliminates bandpass effects in the extinction measurements. Such effects will be illustrated further in Section 4.3. Because the effective 
wavelengths of the broad Johnson filters depend on the shape of a stellar SED (and thus on $E(B-V)$ and $\left.T_{\text {eff }}\right)$, there is no unique transformation between $k(\lambda-V)$ and $k(\lambda-55)$. For our whole sample, which consists — on average — of middle B stars with $E$ $(B-V) \simeq 0.5$, the mean linear transformation is

$$
k(\lambda-55)=\alpha k(\lambda-V)+\beta
$$

where $\alpha=0.990$ and $\beta=0.049$. This shows that the two normalizations are actually quite similar. This relationship implies that

$$
R(55)=\alpha R(V)-\beta .
$$

To determine $k(\lambda-55)$ for a given reddened star, we first produced a fully resolved intrinsic SED $F_{\lambda}$ using the models and stellar properties described in Section 3.1 and covering the complete range of the available data $(\simeq 1150 \AA$ to $2.5 \mu \mathrm{m})$. We then broadened it and resampled the $F_{\lambda}$ to match the various data sets shown in Figure 1. We adopted a $9.8 \AA$ Gaussian (FWHM) for the HST G750L data (Riley et al. 2019) and a $5.5 \AA$ Gaussian (FWHM) for the IUE data (both SW and LW, as based on our own experience with $I U E$ ). In the NIR region, synthetic photometry was performed on the intrinsic SED, as described in Section 2.4, to arrive at intrinsic values for the 2MASS $J, H$, and $K$ magnitudes. The normalized extinction curve was then computed as in Equations (1) and (2).

The normalized optical and NIR extinction curves for all 72 of our program stars are shown in Figure 4. The portions of the curves derived from $2 \mathrm{MASS} J H K$ data are shown as discrete points at $0.46,0.62$, and $0.81 \mu \mathrm{m}^{-1}$, while the portions derived from the combined HST G750L and G430L data are shown from 1.0 to $3.33 \mu \mathrm{m}^{-1}$. For clarity, the stars are sorted by their $K$-band extinction, with the smallest values of $|k(K-55)|$ at the top of panel 4(a) and the largest at the bottom of panel $4(\mathrm{~g})$. The UV portions of the curves for 71 of the 72 program stars have already been presented in F05 and F07, and, apart from the normalization, there are no substantial differences between the results here and those of the earlier work. There is no previously published UV extinction curve for the heavily reddened O star GSC 03712-01870 $[E(B-V) \simeq 1]$, located in the direction of - but beyond-the Perseus arm (Muzzio \& Rydgren 1974). Its far-UV curve (i.e., for $\lambda<2000 \AA$, since no long-wavelength $I U E$ spectra exist) is unremarkable, closely resembling the Milky Way average curve.

A number of the curves in Figure 4 show gaps near $1.2 \mu \mathrm{m}^{-1}$ or $2.7 \mu \mathrm{m}^{-1}$. The SPECTRUM models fail to precisely reproduce the hydrogen lines near the head of the Balmer series (e.g., see Figure 3(g)), which is not surprising given the complexity in modeling the overlapping lines, and this produces "mismatch features" in the curves of the coolest stars in our sample. To avoid these distracting features, we eliminated the region between 3670 and $3760 \AA\left(\sim 2.7 \mu \mathrm{m}^{-1}\right)$ for these stars from the plots. The cool star models also failed to precisely reproduce the higher Paschen lines of hydrogen, so, likewise, we eliminated the region near $8200 \AA$ $\left(\sim 1.2 \mu \mathrm{m}^{-1}\right)$. The TLUSTY OSTAR2002 models used for the hottest of our stars did not include the individual Paschen features, so the regions near these lines were also eliminated. All other features of the curves are on display in the panels of Figure 4. Some discrete stellar mismatch features still exist in some of the curves, but most of the observed structure-from the narrowest to the broadest scales visible on the plots-is a product of the interstellar medium. The most common and prominent stellar mismatch feature arises from nonphotospheric $\mathrm{H} \alpha$ emission, which produces a narrow dip in the curves of some of the stars at $6563 \AA\left(1.52 \mu \mathrm{m}^{-1}\right)$. This feature is labeled (in green) in the top curve of Figure 4(a). Several mismatched stellar He I lines from the $2^{3} P^{0}-n^{3} D$ series are also seen (and labeled in green) in this curve. These absorption lines are stronger in the star (HD 197512) than in the best-fit model and produce narrow spikes in the extinction curve. This is not common in our sample and suggests that HD 197512 is a member of the class of helium-strong B stars as seen in the Orion nebula (e.g., Morgan et al. 1978). Narrow features in the curves arising from DIBs (Herbig 1975) and interstellar Na I and $\mathrm{Ca}$ II absorption are labeled (in red) in panel 4(a) for the HD 14321 curve.

The collection of spectrophotometric extinction curves in Figure 4 exhibits sight line-to-sight line variability on all scales, ranging from the narrow DIBs with widths of a fraction of $1 \mu \mathrm{m}^{-1}$ to the broadest viewable scales. This largest-scale structure can be seen most clearly in a comparison between, for example, the nearly linear curve for HD 210121 in Figure 4(a) and the nearly quadratic curves for HD 294264 or HD 37061 in Figure 4(g). Intermediate-scale structure can also be discerned in many of the curves, notably in HD 197512 or HD 199216 in Figure 4(a). Generally speaking, this structure resembles a broad dip in the extinction at $\sim 1.6 \mu \mathrm{m}^{-1}$ or a rise in extinction at $\sim 2 \mu \mathrm{m}^{-1}$ and corresponds to the Very Broad Structure referred to in Section 1. In a forthcoming paper, Massa et al. (2020) analyze this intermediate-scale structure and its variability and search for relationships with other aspects of NIR-through-UV extinction. In the remainder of this paper, our focus will be on determining the mean properties of NIR-through-UV extinction and quantify the broadest scale of sight line-to-sight line variations.

\section{Results \\ 4.1. The R-dependent Milky Way Extinction Curve}

It was first shown by Cardelli et al. (1989, hereafter CCM) that some large-scale properties of UV extinction (particularly, the general levels in the far-UV) appear correlated with $R(V)$. This wavelength coherence allows a family of $R(V)$-dependent extinction curves to be derived, potentially reducing the uncertainties in applying extinction corrections to sight lines with measured values of $R(V)$ but otherwise unknown extinction properties. Subsequent studies (e.g., F07) have shown that much of this correlated behavior is driven by a relatively small number of sight lines with extreme values of $R(V)$ and that extinction curves with similar values of $R(V)$ may have a wide range in UV properties (e.g., Mathis \& Cardelli 1992; Valencic et al. 2004). Nevertheless, the $R(V)$-dependence is important because, with appropriate estimation of the uncertainties, it can (1) potentially reduce dereddening errors, (2) allow a meaningful definition of a Milky Way average extinction curve, as that which corresponds to the mean observed value of $R(V)$, and (3) provide insight into the link between interstellar environment and dust grain properties (see the discussion in Fitzpatrick 1999).

We examined the $R$-dependence of the curves in our sample in the simplest way, by plotting the values $k(\lambda-55)$ versus $R$ at each wavelength point in the data set. We determined values of $R$ by using the results from F07, who found that-when fitting the NIR data with a power-law formula-the values of 


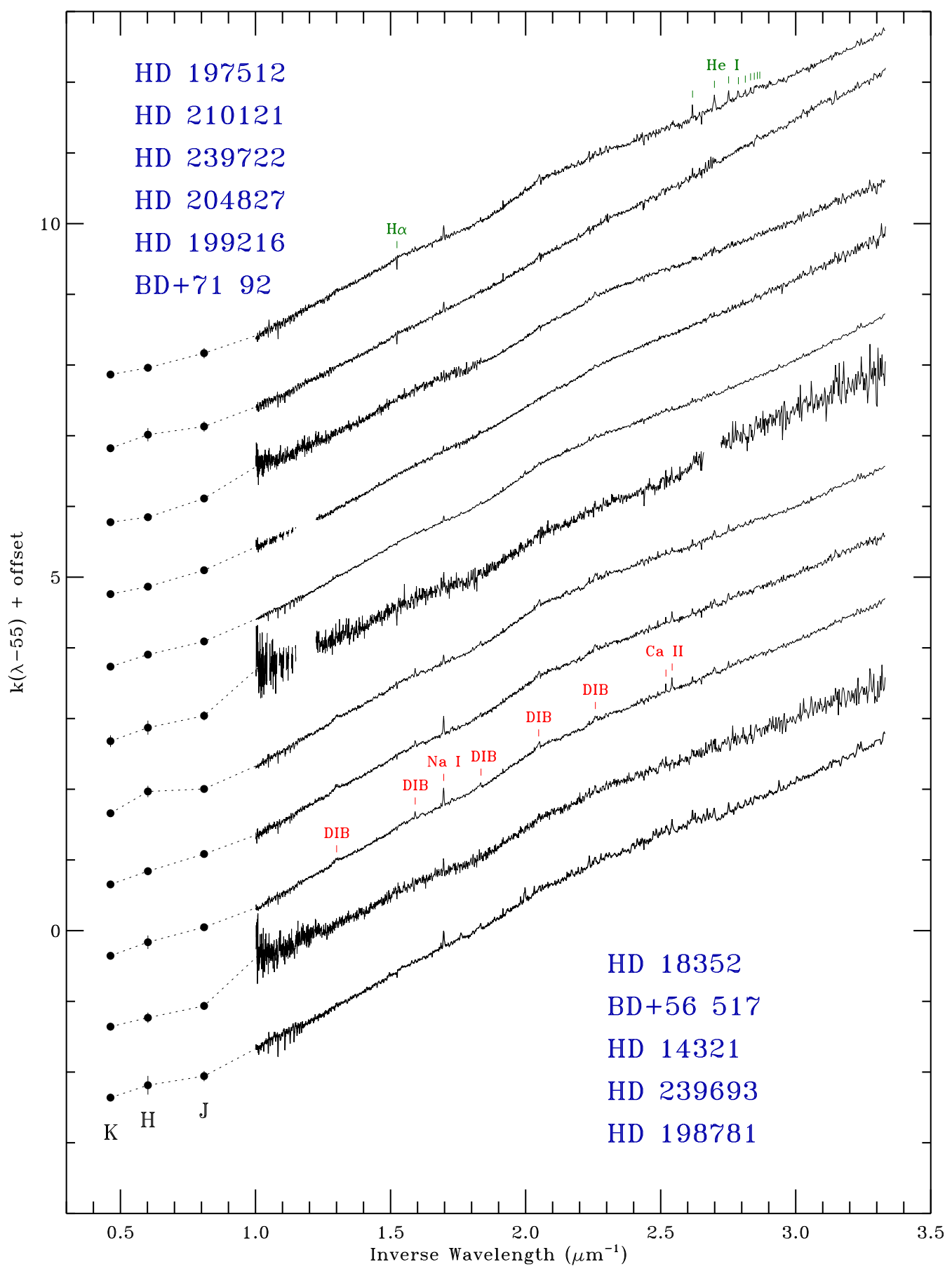

Figure 4. Normalized NIR-through-optical extinction curves for the program stars, in the form $k(\lambda-55) \equiv E(\lambda-55) / E(44-55)$. The large filled circles indicate the extinction in the 2MASS JHK bands. The solid curves show the spectrophotometric extinction curves derived from the HST G430L and G750L data, which are joined at $5450 \AA$. Narrow features in the curves resulting from stellar spectral mismatch are labeled in green. Interstellar features, including gas-phase absorption lines and DIBs, are labeled in red. Gaps appear in some of the curves near the head of the Paschen series $\left(\lambda^{-1} \simeq 1.2 \mu \mathrm{m}^{-1}\right)$ and the head of the Balmer series $\left(\lambda^{-1} \simeq 2.7 \mu \mathrm{m}^{-1}\right)$, where stellar mismatch features were removed. See the discussion in Section 3.2. The curves are organized in order of increasing $|k(K-55)|$ throughout the figures. Arbitrary offsets are added for clarity.

(The complete figure set (seven images) is available.)

$R(V)$ were related to the extinction at the $K$ band by the relation

$$
R(V)=-1.19 \times k(K-V)-0.26 .
$$

This can be converted to our normalization system by using the mean transformation in Equation (5) and yields

$$
R(55)=-1.19 \times k(K-55)-0.25 .
$$

Plots of $k(\lambda-55)$ versus $R(55)$ are shown in Figure 5 for 10 representative wavelengths (out of a total of 2700 points per curve), spanning the NIR-through-UV region. The $R(55)$ dependence is noisy but reasonably well defined for most data points. To quantify the relationship, we fit a simple linear function at each wavelength, recording the intercept, slope, and 

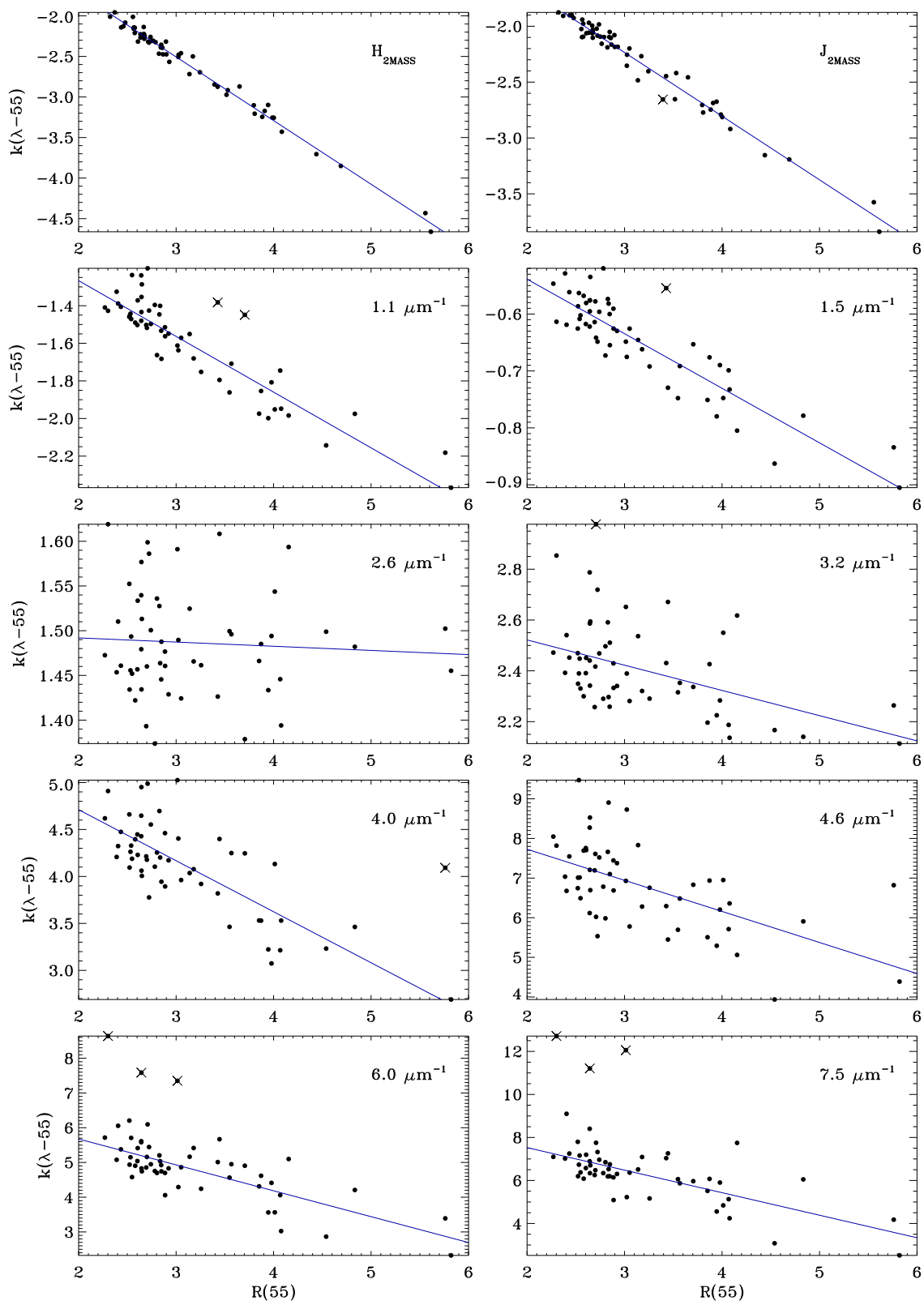

Figure 5. Examples of the $R(55)$-dependence of the normalized extinction curves, where $R(55) \equiv A(55) / E(44-55)$, for the 55 stars in our sample with $E(B-V)>0.30$ (solid symbols). The specific wavelengths shown include the NIR $\left(H, J\right.$, and $\left.1.1 \mu \mathrm{m}^{-1}\right)$, optical $\left(1.5,2.6\right.$, and $\left.3.2 \mu \mathrm{m}^{-1}\right)$, and UV $(4.0,4.6,6.0$, and $\left.7.5 \mu \mathrm{m}^{-1}\right)$ spectral regions. The values of $R(55)$ for each star were determined from Equation (7), as discussed in Section 4.1. The blue lines show linear fits to $k(\lambda-55)$ versus $R(55)$ at each wavelength. Crossed-out points were excluded from the fit using a "sigma-clipping" criterion set at $2.5 \sigma$. Fits such as these were performed for each of the 2700 data points defining the full extinction curves.

standard deviation about the fit. A single-iteration $\sigma$ clip with a threshold set at $2.5 \sigma$ was performed to eliminate extreme points from the fit. In Figure 5, the blue lines show the fits, and points that are crossed out indicate those rejected by the $\sigma$ clipping. This procedure was restricted to the 55 sight lines in our sample for which $E(B-V)>0.30$, which minimizes the impact of the larger random errors inherent in the low $E(B-V)$ curves.

Figure 6 graphically illustrates the overall results of fitting the linear $R$-dependence in our data set. The curve in the top panel shows $k(\lambda-55)_{0}$, the values of the fits at $R(55)=$ 3.02 , which corresponds to the Milky Way mean value of $R(V)=3.10$ (see Equation (5)). Thus, this curve shows our measurement of the extinction representative of the diffuse ISM over the wavelength range $1150 \AA$ to $2.5 \mu \mathrm{m}$. Prominent stellar and interstellar mismatch features are labeled in blue. The middle panel shows the slopes of the fits, $s(\lambda-55) \equiv \Delta k$ $(\lambda-55) / \Delta R(55)$, illustrating the $R(55)$-dependence. The coherence and magnitude of the structure in the $s(\lambda-55)$ plot, as compared to its point-to-point scatter, indicate that the fitting procedure has identified a true $R$-dependence in the data, rather than just quantifying the noise. Finally, the bottom panel shows $\sigma[k(\lambda-55)]$, the standard deviation of the individual sight lines against the mean relationship, which quantifies the combination of measurement noise and real deviations from the $R$-dependent relation.

A comparison between the functional dependence of extinction on $R$ adopted here and that used by CCM is given in Appendix B. 

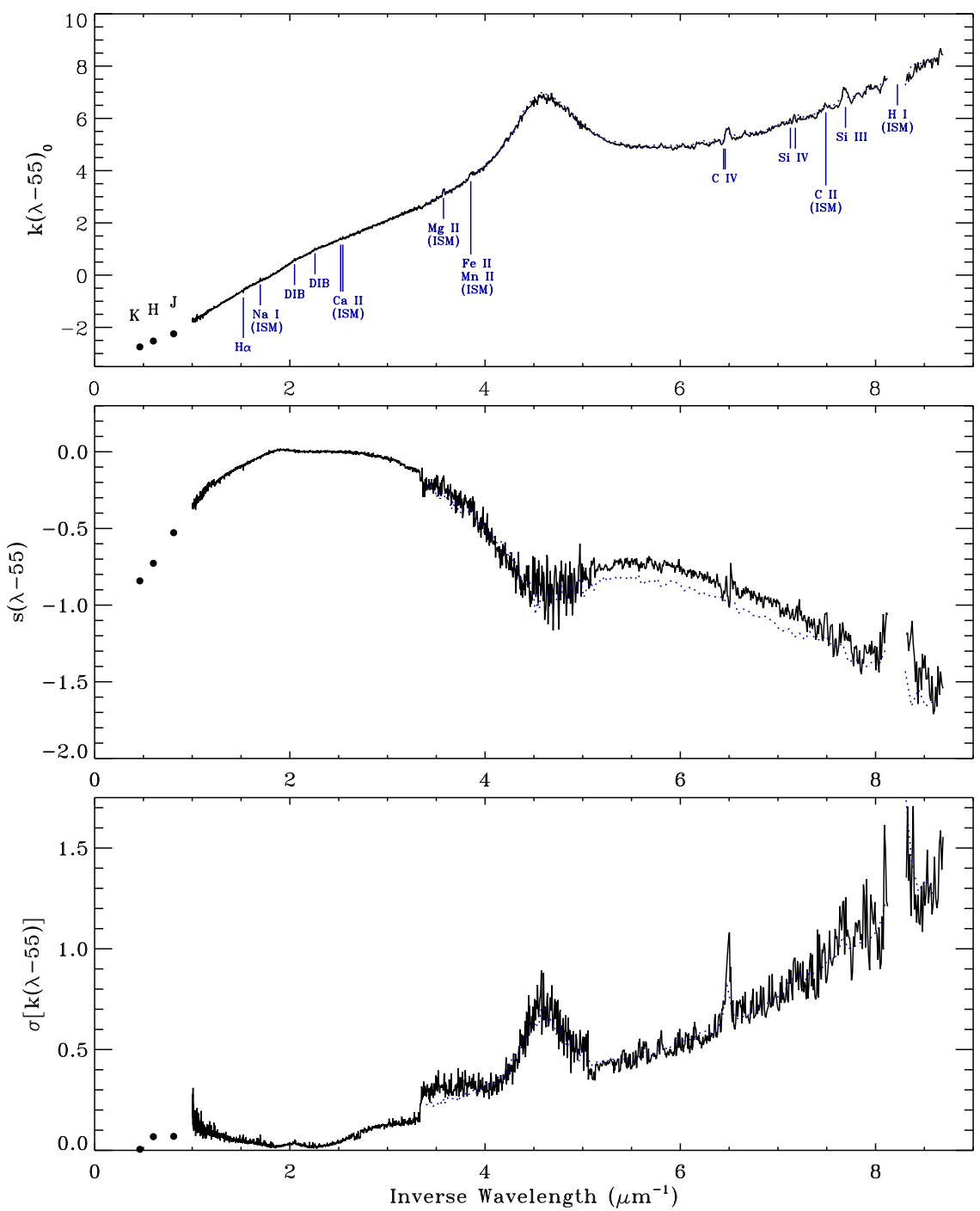

Figure 6. The $R(55)$-dependent relationship. Top panel: mean NIR-through-UV extinction curve in the form $k(\lambda-55)_{0} \equiv E(\lambda-55) / E(44-55)$ for the case of $R(55)=3.02$, as derived from the linear fits to $k(\lambda-55)$ versus $R(55)$ described in Section 4.1 and illustrated in Figure 5. This value of $R(55)$ corresponds to $R(V)=3.10$, which is generally considered to be the Milky Way mean. Features in the UV curve due to stellar mismatch, interstellar gas absorption lines, or geocoronal Ly $\alpha$ emission are indicated. Middle panel: linear dependence of the mean curve on $R(55)$. These values are the slopes of the linear fits described in Section 4.1. Bottom panel: standard deviation of the individual curves about the mean $R(55)$-dependence. The dotted blue curves in the UV region of each panel show the results of a similar analysis performed by us on the much larger set of UV extinction curves presented by F07; see the discussion in Section 5.1.

\subsection{A Tabular Form of the R-dependent Relationship}

To make the new results in Figure 6 useful for other extinction investigations, we created a tabular form of these data. This was done by fitting the three curves in Figure 6 with smooth functions and then recording the values of the functions at selected wavelengths. The details of this process are illustrated in Figure 7, where the smooth blue curves show the fits to the data and the blue circles indicate the selected points.

In the optical/NIR, that is, for the HST spectrophotometric data, we fit cubic splines to the three curves, with anchor points spaced every $0.1 \mu \mathrm{m}^{-1}$ in the interval $1.0 \mu \mathrm{m}^{-1}(10000 \AA)$ to $3.3 \mu \mathrm{m}^{-1}(3030 \AA)$, with intermediate points added between 1.2 and $2.9 \mu \mathrm{m}^{-1}$ and two special points-at $1.818 \mu \mathrm{m}^{-1}(5500 \AA)$ and $2.273 \mu \mathrm{m}^{-1}(4400 \AA)$ —added to enforce the normalization. Regions near the Balmer and Paschen jumps that are affected by spectral mismatch were given zero weight so that the fits passed smoothly through these regions in the $s(\lambda-V)$ and $\sigma[k(\lambda-55)]$ curves. Likewise, data affected by interstellar absorption features (including DIBs) and stellar mismatch features were given zero weight. Notice that the values of $\sigma[k(\lambda-55)]$ do not go to zero at the normalization points. This is because (as noted in Section 3.2) the normalization was performed using interpolation within a bin containing the normalization wavelengths. Thus, these small values of $\sigma[k(\lambda-55)] \simeq 0.02$ reflect the point-topoint scatter in the normalization regions of the curves, rather than sight line-to-sight line variations. The large values of $\sigma[k(\lambda-55)]$ at the longest optical wavelengths are likely dominated by statistical noise in the HST G750L data rather than curve-to-curve variability. The true curve-to-curve scatter is probably at the level of $\sigma[k(\lambda-55)] \simeq 0.1$, similar to the shorter wavelength G750L data and the 2MASS $J$ and $H$ values.

In the UV, we used variations of the F07 fitting function to achieve smooth representations of the curves. The fits were performed over the wavelength range of the IUE data, $3.3 \mu \mathrm{m}^{-1}$ $(3030 \AA)$ to $8.7 \mu \mathrm{m}^{-1}(1150 \AA)$, plus a small section of the $H S T$ data, from 3.0 to $3.3 \mu \mathrm{m}^{-1}$, which was included to ensure continuity with the optical region. Spectral mismatch features 

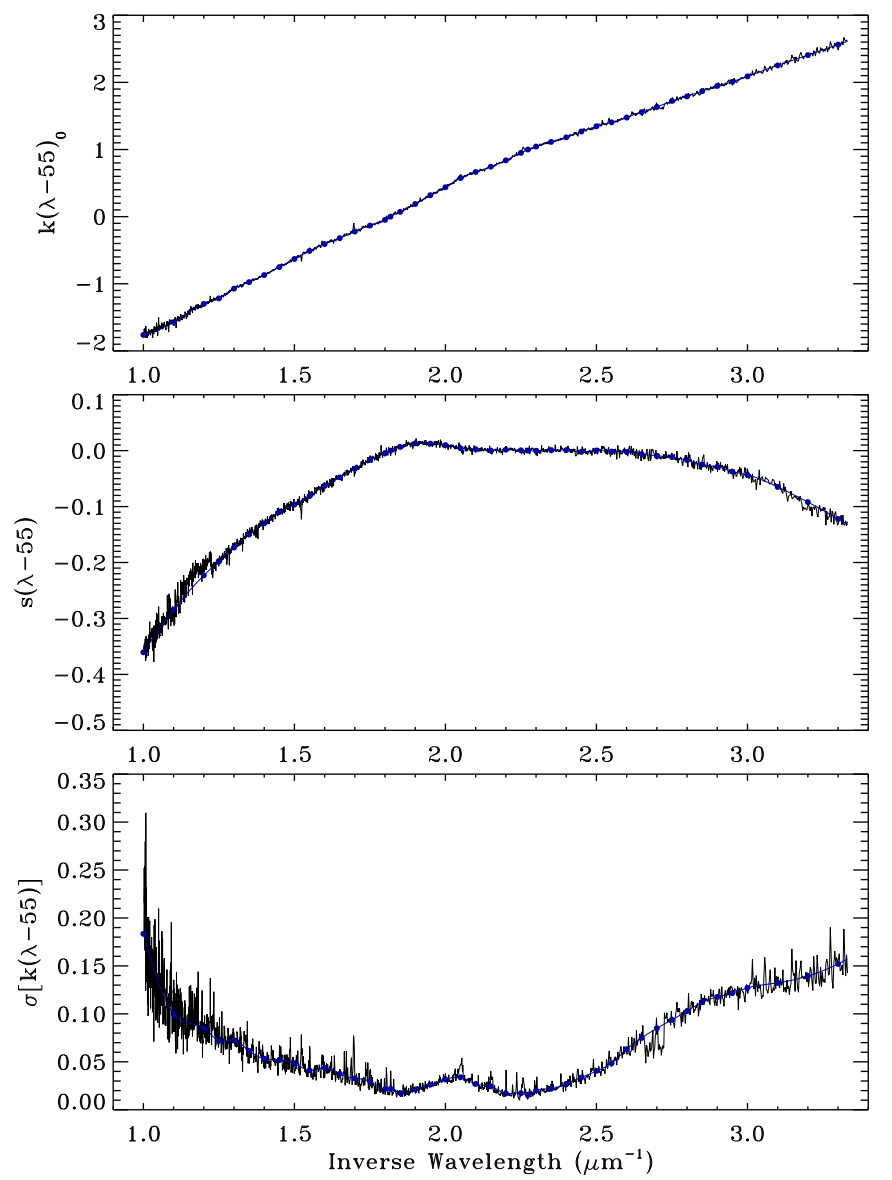
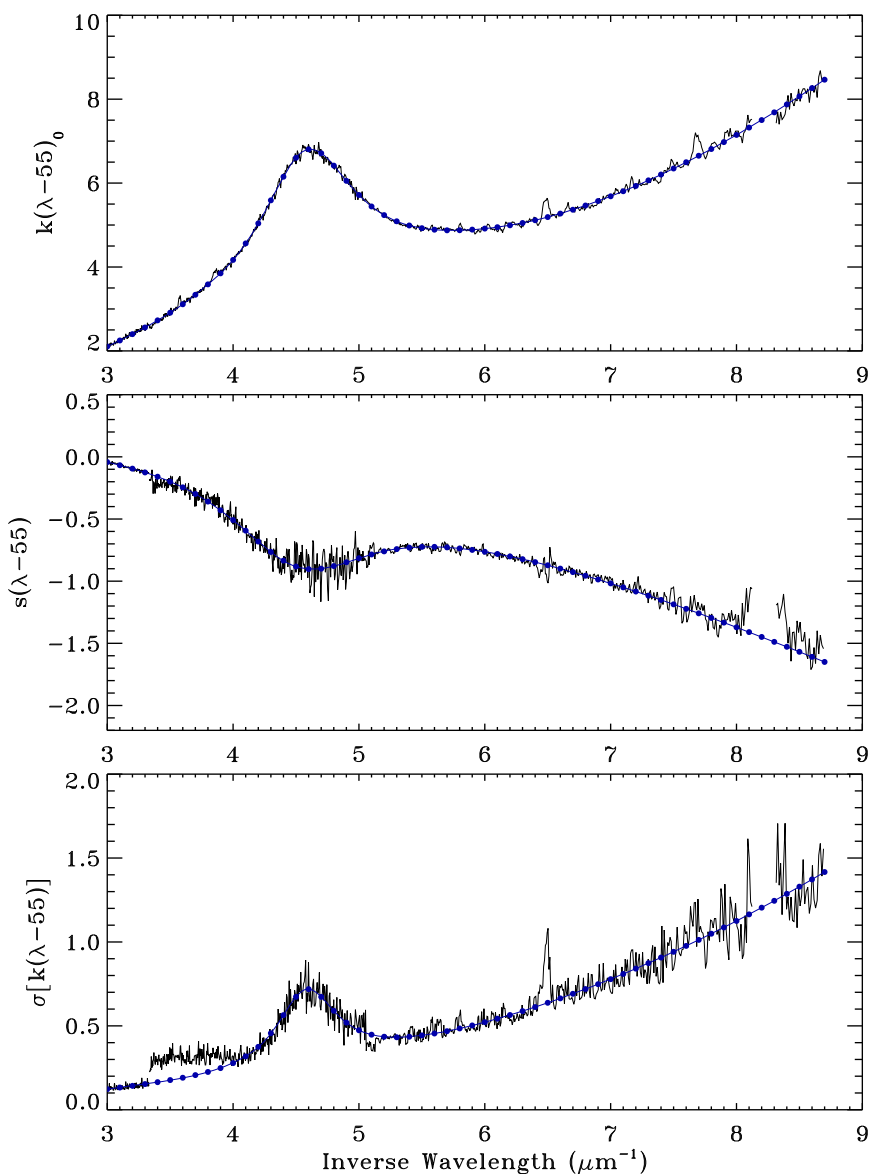

Figure 7. Creating a tabular form of the $R$-dependence of the Milky Way extinction curve in the optical/NIR (left) and UV (right) spectral regions. In the optical, a cubic spline was fit to the $k(\lambda-55)_{0}$ (top panel), $s(\lambda-55)$ (middle panel), and $\sigma[k(\lambda-55)]$ (bottom panel) curves. The anchor points were spaced at $0.05 \mu \mathrm{m}^{-1}$ intervals between 1.2 and $3.0 \mu \mathrm{m}^{-1}$ and at intervals of $0.1 \mu \mathrm{m}^{-1}$ outside that range. The values of the anchors were adjusted iteratively to achieve the best fit to the respective curves. Additional points were added at $1.818 \mu \mathrm{m}^{-1}(5500 \AA)$ and $2.273 \mu \mathrm{m}^{-1}$ (4400 $\AA$ ) to ensure the normalization. The cubic spline fits are shown by the blue curves, and the anchor points are indicated by the filled blue circles. In the UV, the data were fit by variants of the UV fitting functions adopted by F07. The fits are shown by the blue curves, and their values at $0.1 \mu \mathrm{m}^{-1}$ intervals are shown by the filled blue circles. To ensure continuity with the optical results, the UV fits were extended into the optical region (between 3.0 and $3.3 \mu \mathrm{m}^{-1}$ ), and low weight was given to the longest-wavelength UV data, due to instability in the calibration of IUE's long-wavelength camera at these wavelengths. A tabular form of these curves is given in Table 3. See the discussion in Section 4.2.

were given zero weight in the fits. Figures 6 and 7 show that there is a significant discontinuity between the longest UV wavelengths and the shortest optical wavelengths in the $\sigma[k(\lambda-55)]$ curves. We attribute this to instability in the calibration of IUE's LWR and LWP cameras at their longest wavelengths, where the camera sensitivities are highly dependent on the placement of the spectra on the camera face. We gave the longest UV wavelengths zero weight so that the fits would join smoothly with the optical HST data. There is also a small discontinuity noticeable in $\sigma[k(\lambda-55)]$ at the joint between IUE's short- and long-wavelength cameras at $5.05 \mu \mathrm{m}^{-1}(1980 \AA)$, where, once again, the extreme sensitivity of the long-wavelength cameras to spectrum placement (and the lower general sensitivity of the cameras) manifests as enhanced scatter. Values of the fits at $0.1 \mu \mathrm{m}^{-1}$ intervals over the range $3.3-8.7 \mu \mathrm{m}^{-1}$, as indicated by the filled circles in Figure 7, were recorded.

The final tabular form of our extinction results is given in Table 3. The columns in the table list the wavelengths (in $\left.\mu \mathrm{m}^{-1}\right)$, followed by the values of $k(\lambda-55)_{0}, s(\lambda-55)$, and $\sigma[k(\lambda-55)]$. For the spectrophotometric data, these all correspond to the filled circles in Figure 7. For the 2MASS $J H K$ data, the values in the table are simply those shown in Figure 6. The result that $\sigma[k(K-55)]=0$ arises because our values of $R(55)$ are tied directly to the $K$-band measurement via Equation (7).

The data in Table 3 can be used to reconstruct an extinction curve in the form of $k(\lambda-55)$ over the indicated wavelength range for any value of $R(55)$ or $R(V)$ ranging from 2.5 to 6.0. The restriction on the range of valid $R$ values comes directly from the range of $R$ in our data (see Figure 5). The curve can be constructed using the formula

$$
\begin{aligned}
k(\lambda-55)_{R(55)}= & k(\lambda-55)_{0}+s(\lambda-55) \\
& \times\left[R(55)-R(55)_{0}\right]
\end{aligned}
$$

where $R(55)_{0}=3.02$ and $k(\lambda-55)_{0}$ is the extinction for the case $R(55)=R(55)_{0}$. If $R$ is expressed in $R(V)$ notation, then Equation (8) transforms to

$$
\begin{aligned}
k(\lambda-55)_{R(V)}= & k(\lambda-55)_{0}+s(\lambda-55) \\
& \times[R(V)-3.10] \times 0.99 .
\end{aligned}
$$

Four examples of extinction curves constructed from Table 3 are shown in Figure 8, for values of $R(V)=2.5,3.1,4.5$, and 6.0. The $R$-dependent extinction relationship is available in the dust_extinction Python package ${ }^{10}$ as the "F20" model.

\footnotetext{
${ }^{10}$ https://dust-extinction.readthedocs.io/
} 
Table 3

Average Milky Way Extinction Curve

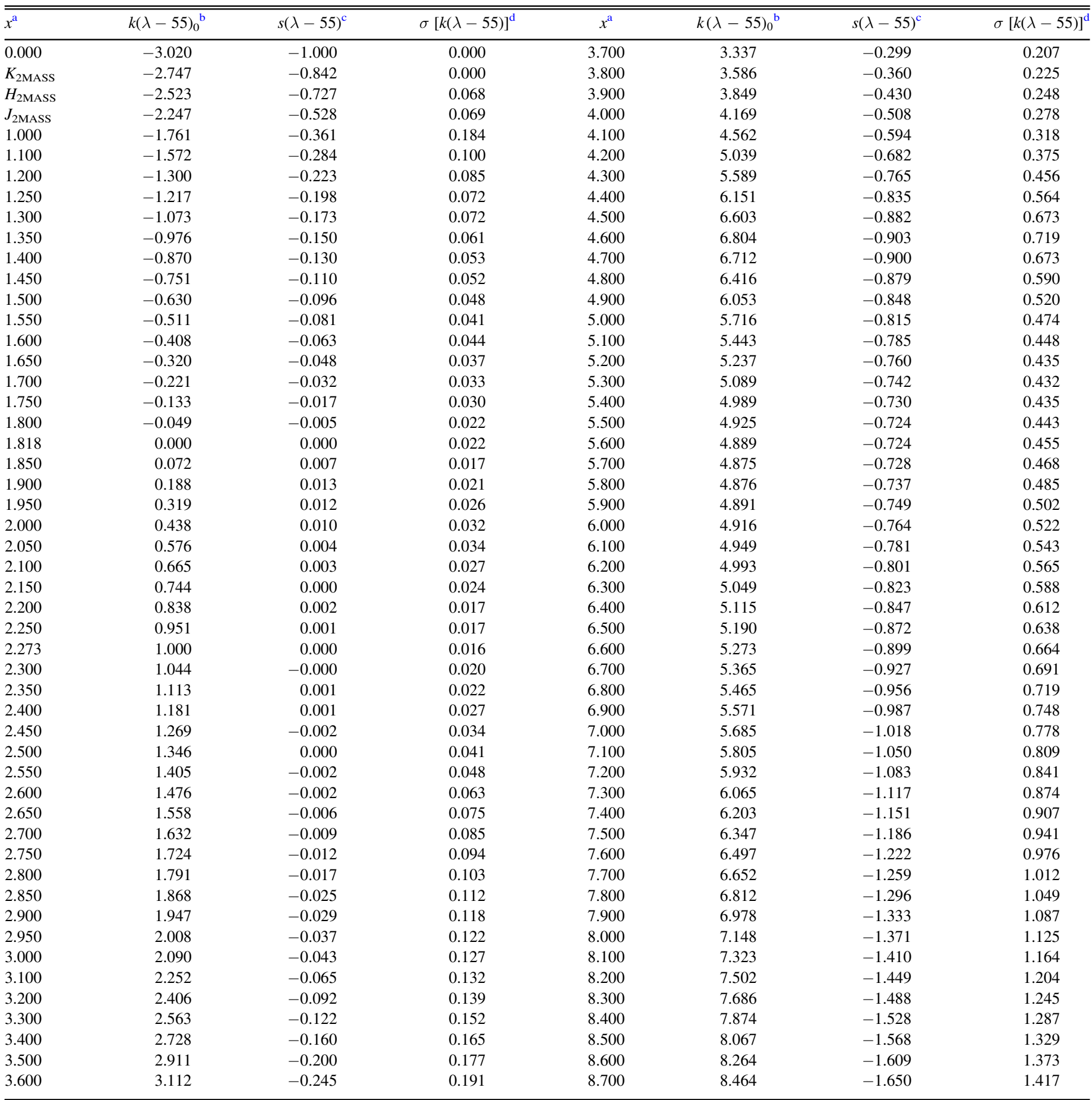

Notes.

a Inverse wavelength in units of $\mu \mathrm{m}^{-1}$.

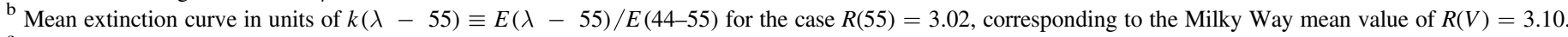

${ }^{\mathrm{c}}$ Linear dependence of $k(\lambda-55)$ on $R(55)$ over the range $2.5 \leqslant R(55) \leqslant 6.0$.

${ }^{\mathrm{d}}$ Curve-to-curve standard deviation as a function of wavelength.

\subsection{Reddening Slopes for Optical Photometry}

Given the advent of large ground-based surveys and the overall prevalence of the data, optical photometry remains a powerful astronomical tool. Knowledge of the effects of interstellar extinction on the various photometric systems is important for disentangling the intrinsic and extrinsic (i.e., interstellar) contributions to photometric measurements.

To illustrate and quantify the effects of interstellar extinction on optical SEDs, we have computed the photometric reddening slopes predicted by our new optical extinction curve for three common photometric systems: Johnson $U B V$, Strömgren uvby, 


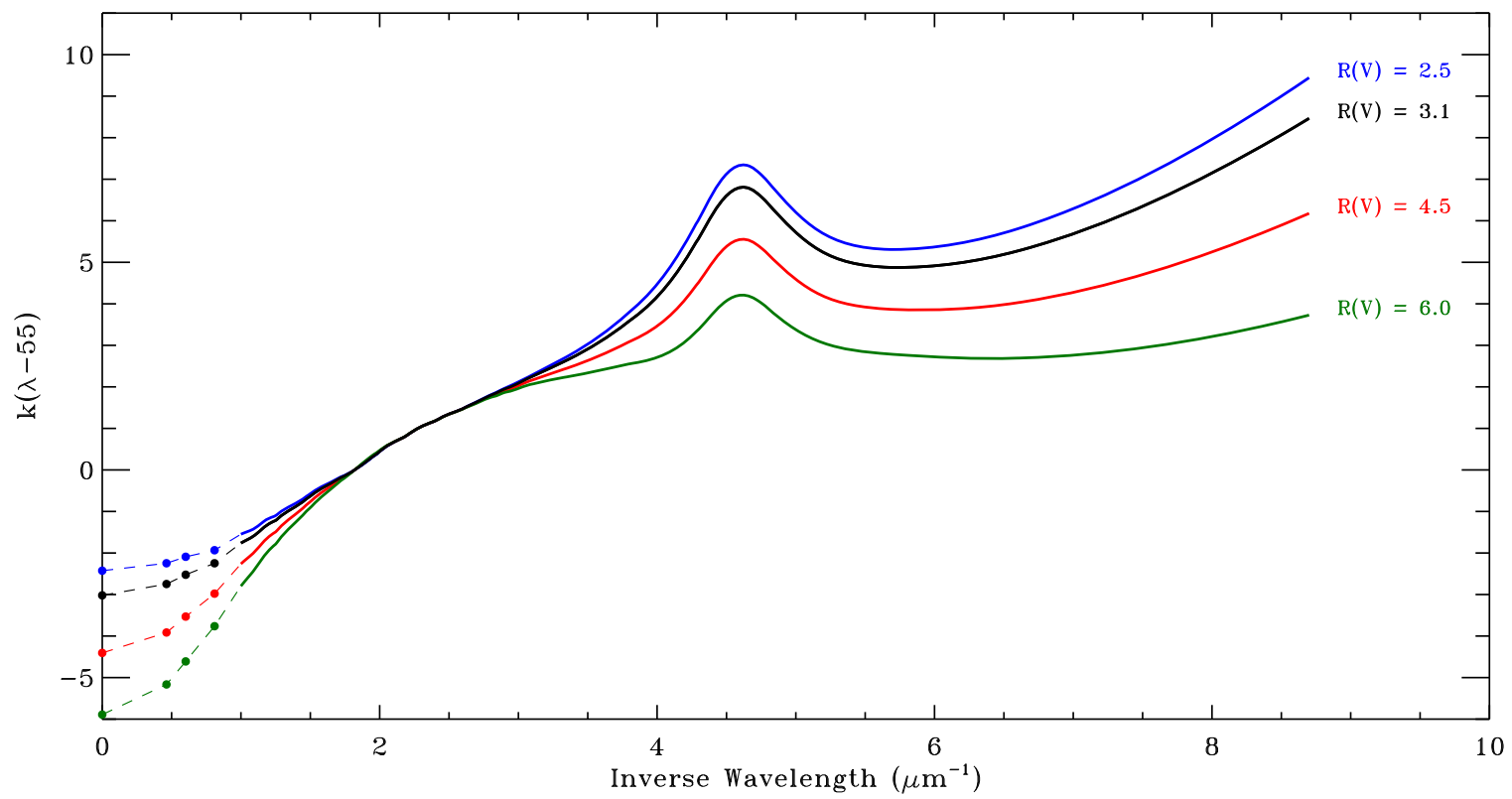

Figure 8. $R(V)$-dependent NIR-through-UV extinction curves from this study, shown for several values of $R(V)$. The curves were produced from the data in Table 3 using Equation (9).

and Sloan Digital Sky Survey ugriz. The slopes were determined by performing synthetic photometry on intrinsic and reddened stellar energy distributions, using the ATLAS9 atmosphere models, and then forming the various differences and ratios. The results are presented in Tables 4 and 5 . The measurements were made for two values of reddening $[E(44-55)=0.50$ and 1.0], four values of $R(V)\left(2.5,3.1,4.5\right.$, and 6.0), and 10 values of $T_{\text {eff }}$ (from 7000 to $35,000 \mathrm{~K})$. The synthetic Johnson and Strömgren photometry was performed as described by Fitzpatrick \& Massa (2005b). We modified the calibration of the synthetic values, however, to take advantage of the new HST G430L and G750L observations obtained for this program. In brief, we computed synthetic, uncalibrated Johnson and Strömgren photometric indices $(B-V$, $U-B, b-y, m_{1}$, and $c_{1}$ ) using the $H S T$ spectrophotometry for each of our 72 reddened stars, plus three additional unreddened stars HD 38666, HD 34816, and HD 214680 from the HST archives. We then determined the linear transformation between these synthetic values and the observed photometric indices. The results are similar to those reported in Fitzpatrick \& Massa (2005b), and the scatter of the observed values about the synthetic results is consistent with the expected observational error. The synthetic ugriz photometry was performed as described in Casagrande \& VandenBerg (2014), with filter profiles from Doi et al. (2010).

The $E(B-V) / E(44-55)$ column in Table 4 illustrates the uncertainty in measuring the "amount" of extinction using broadband photometry. For a fixed extinction [as measured by $E(44-55)]$, the observed values of $E(B-V)$ can vary significantly depending on the stellar $T_{\text {eff }}$, reflecting variations in the effective wavelengths of the broad $B$ and $V$ filters. For the same reason, the values of $E(B-V) / E(44-55)$ also depend on the overall level of extinction. The Johnson and Strömgren reddening slopes implied by the $R(V)=3.1$ curve are-with the exception of $E\left(c_{1}\right)$ / $E(B-V)$-consistent with the accepted values (see Fitzpatrick 1999), including the $E(B-V)$-dependence of $E(U-B) / E(B-V)$ (e.g., Fitzgerald 1970). The accepted $E\left(c_{1}\right) / E(B-V)$ ratio $(\simeq 0.20)$ is from Crawford (1975) and based on an "eye fit" to photometry of 50 O-type stars. However, it is clear from Crawford's Figure 5 that a steeper ratio is needed to explain the most heavily reddened $\mathrm{O}$ stars in the sample. In addition, Crawford notes that a preliminary study of B-type stars yields a larger value $(\simeq 0.24)$. We suggest that the results in Table 4 present the most reliable estimates for the Strömgren reddening slopes. Note also that, while the intermediate-bandwidth Strömgren slopes are relatively insensitive to effective wavelength shifts [i.e., minimal dependence on $T_{\text {eff }}$ or $E(44-55)$ ], the $E\left(c_{1}\right)$ ratio is strongly dependent on $R(V)$ due to the short-wavelength $u$ filter. The same sensitivity is also seen in the $E(U-B)$ slope, potentially allowing both indices to be used as diagnostics of $R(V)$.

Qualitatively similar effects, in terms of $T_{\text {eff }}$ and $E(44-55)$ sensitivity, can be seen in the ugriz results in Table 5. Note that the reddening slopes involving the longer-wavelength $\mathrm{riz}$ filters $\left(\lambda_{\text {eff }}=6231,7625\right.$, and $9134 \AA$, respectively) are particularly sensitive to the values of $R(V)$. Given that there is less curveto-curve scatter relative to the $R$-correlation at these longer wavelengths (see Figure 7), as compared to that for the Strömgren $u$ or Johnson $U$ filters, these filters could provide a very strong diagnostic of $R(V)$.

\subsection{Balmer Decrement}

The formation rate of massive stars in external galaxies can be inferred from the luminosities of emission lines-notably, the Balmer lines-arising in the galaxies' H II regions (e.g., Kennicutt 1983; Gallagher et al. 1984). This requires that the line emission first be corrected for the attenuating effects of interstellar extinction. A common technique is to compare the observed luminosity ratio of two lines, such as $\mathrm{H} \alpha / \mathrm{H} \beta$, to the expected intrinsic ratio and interpret any difference as a result of the wavelength-dependent effects of reddening. This is the "Balmer decrement" method and allows an estimate of $E(B-V)$, which further allows an estimate of the total extinction at the line wavelengths (e.g., Calzetti et al. 1994). This process requires an understanding of the intrinsic emission mechanism and, apropos to this paper, a well-determined interstellar extinction law.

Using the results in Table 3 and following the derivation in, for example, Section 3 of Domínguez et al. (2013), we can compute the implied reddening based on any pair of lines for 
Table 4

$U B V$ and $u v b y$ Extinction Indices

\begin{tabular}{|c|c|c|c|c|c|c|c|c|c|c|}
\hline$T_{\text {eff }}$ & $\frac{E(B-V)}{E(44-55)}$ & $\begin{array}{l}\frac{E(U-B)}{E(B-V)} \\
\end{array}$ & 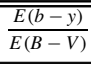 & $\begin{array}{c}\frac{E\left(m_{1}\right)}{E(b-y)} \\
\end{array}$ & $\frac{E\left(c_{1}\right)}{E(b-y)}$ & $\frac{E(B-V)}{E(44-55)}$ & $\begin{array}{l}\frac{E(U-B)}{E(B-V)} \\
\end{array}$ & $\frac{E(b-y)}{E(B-V)}$ & $\begin{array}{c}\frac{E\left(m_{1}\right)}{E(b-y)} \\
\end{array}$ & $\frac{E\left(c_{1}\right)}{E(b-y)}$ \\
\hline & \multicolumn{10}{|c|}{$R(V)=2.5$} \\
\hline & \multicolumn{5}{|c|}{$E(44-55)=0.50$} & \multicolumn{5}{|c|}{$E(44-55)=1.00$} \\
\hline $7000 \mathrm{~K}$ & 0.945 & 0.806 & 0.752 & -0.299 & 0.285 & 0.933 & 0.830 & 0.762 & -0.299 & 0.279 \\
\hline $8000 \mathrm{~K}$ & 0.955 & 0.760 & 0.746 & -0.300 & 0.289 & 0.943 & 0.782 & 0.755 & -0.301 & 0.283 \\
\hline $9000 \mathrm{~K}$ & 0.964 & 0.730 & 0.738 & -0.300 & 0.290 & 0.953 & 0.750 & 0.747 & -0.300 & 0.283 \\
\hline $10000 \mathrm{~K}$ & 0.972 & 0.718 & 0.732 & -0.297 & 0.290 & 0.961 & 0.739 & 0.740 & -0.298 & 0.283 \\
\hline $12500 \mathrm{~K}$ & 0.981 & 0.736 & 0.725 & -0.295 & 0.293 & 0.970 & 0.755 & 0.733 & -0.295 & 0.287 \\
\hline $15000 \mathrm{~K}$ & 0.985 & 0.752 & 0.722 & -0.294 & 0.296 & 0.974 & 0.771 & 0.730 & -0.295 & 0.290 \\
\hline $20000 \mathrm{~K}$ & 0.990 & 0.765 & 0.718 & -0.293 & 0.299 & 0.979 & 0.784 & 0.726 & -0.294 & 0.293 \\
\hline $25000 \mathrm{~K}$ & 0.993 & 0.773 & 0.716 & -0.292 & 0.300 & 0.982 & 0.791 & 0.724 & -0.292 & 0.294 \\
\hline $30000 \mathrm{~K}$ & 0.996 & 0.775 & 0.713 & -0.291 & 0.301 & 0.985 & 0.794 & 0.722 & -0.291 & 0.295 \\
\hline $35000 \mathrm{~K}$ & 0.997 & 0.778 & 0.712 & -0.290 & 0.301 & 0.986 & 0.796 & 0.721 & -0.291 & 0.295 \\
\hline
\end{tabular}

\begin{tabular}{|c|c|c|c|c|c|c|c|c|c|c|}
\hline \multirow[b]{2}{*}{$7000 \mathrm{~K}$} & \multicolumn{5}{|c|}{$E(44-55)=0.50$} & \multicolumn{5}{|c|}{$E(44-55)=1.00$} \\
\hline & 0.951 & 0.793 & 0.748 & -0.301 & 0.263 & 0.940 & 0.815 & 0.756 & -0.301 & 0.258 \\
\hline $8000 \mathrm{~K}$ & 0.959 & 0.748 & 0.742 & -0.302 & 0.266 & 0.949 & 0.769 & 0.750 & -0.303 & 0.261 \\
\hline $9000 \mathrm{~K}$ & 0.968 & 0.718 & 0.735 & -0.301 & 0.267 & 0.959 & 0.738 & 0.743 & -0.302 & 0.262 \\
\hline $10000 \mathrm{~K}$ & 0.976 & 0.707 & 0.729 & -0.299 & 0.267 & 0.966 & 0.727 & 0.736 & -0.300 & 0.261 \\
\hline $12500 \mathrm{~K}$ & 0.985 & 0.724 & 0.722 & -0.297 & 0.270 & 0.975 & 0.743 & 0.730 & -0.297 & 0.264 \\
\hline $15000 \mathrm{~K}$ & 0.989 & 0.739 & 0.719 & -0.296 & 0.273 & 0.979 & 0.758 & 0.727 & -0.296 & 0.267 \\
\hline $20000 \mathrm{~K}$ & 0.994 & 0.752 & 0.716 & -0.295 & 0.276 & 0.984 & 0.770 & 0.723 & -0.295 & 0.270 \\
\hline $25000 \mathrm{~K}$ & 0.997 & 0.759 & 0.713 & -0.293 & 0.276 & 0.987 & 0.777 & 0.720 & -0.294 & 0.271 \\
\hline $30000 \mathrm{~K}$ & 0.999 & 0.761 & 0.711 & -0.293 & 0.277 & 0.990 & 0.779 & 0.718 & -0.293 & 0.271 \\
\hline $35000 \mathrm{~K}$ & 1.001 & 0.764 & 0.710 & -0.292 & 0.277 & 0.991 & 0.782 & 0.717 & -0.292 & 0.272 \\
\hline
\end{tabular}

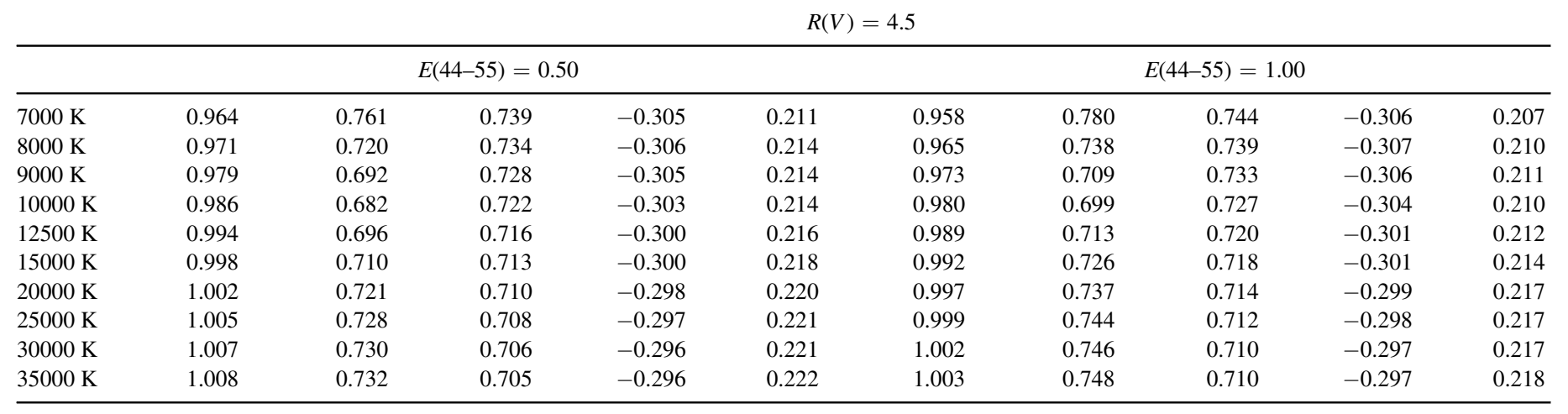

\begin{tabular}{|c|c|c|c|c|c|c|c|c|c|c|}
\hline \multirow[b]{2}{*}{$7000 \mathrm{~K}$} & \multicolumn{5}{|c|}{$E(44-55)=0.50$} & \multicolumn{5}{|c|}{$E(44-55)=1.00$} \\
\hline & 0.978 & 0.728 & 0.729 & -0.309 & 0.155 & 0.979 & 0.743 & 0.729 & -0.310 & 0.153 \\
\hline $8000 \mathrm{~K}$ & 0.983 & 0.690 & 0.725 & -0.310 & 0.158 & 0.983 & 0.705 & 0.726 & -0.312 & 0.155 \\
\hline $9000 \mathrm{~K}$ & 0.990 & 0.664 & 0.720 & -0.309 & 0.158 & 0.990 & 0.678 & 0.721 & -0.311 & 0.155 \\
\hline $10000 \mathrm{~K}$ & 0.997 & 0.654 & 0.715 & -0.307 & 0.156 & 0.997 & 0.668 & 0.716 & -0.309 & 0.154 \\
\hline $12500 \mathrm{~K}$ & 1.004 & 0.667 & 0.709 & -0.305 & 0.158 & 1.004 & 0.681 & 0.710 & -0.306 & 0.155 \\
\hline $15000 \mathrm{~K}$ & 1.008 & 0.679 & 0.707 & -0.304 & 0.159 & 1.008 & 0.692 & 0.708 & -0.305 & 0.157 \\
\hline $20000 \mathrm{~K}$ & 1.012 & 0.688 & 0.704 & -0.303 & 0.161 & 1.011 & 0.702 & 0.705 & -0.304 & 0.159 \\
\hline $25000 \mathrm{~K}$ & 1.014 & 0.694 & 0.702 & -0.301 & 0.161 & 1.014 & 0.708 & 0.703 & -0.303 & 0.159 \\
\hline $30000 \mathrm{~K}$ & 1.016 & 0.695 & 0.700 & -0.301 & 0.161 & 1.016 & 0.709 & 0.701 & -0.302 & 0.159 \\
\hline $35000 \mathrm{~K}$ & 1.017 & 0.697 & 0.700 & -0.300 & 0.161 & 1.017 & 0.711 & 0.701 & -0.301 & 0.159 \\
\hline
\end{tabular}

which the intrinsic ratio is known. Two examples are

$$
\begin{aligned}
E(44-55)= & \frac{2.5}{1.16+0.09 \times\left(R_{55}-3.02\right)} \\
& \times \log _{10}\left[\frac{(\mathrm{H} \alpha / \mathrm{H} \beta)_{\mathrm{obs}}}{(\mathrm{H} \alpha / \mathrm{H} \beta)_{\mathrm{int}}}\right]
\end{aligned}
$$

and

$$
E(44-55)=-5.43 \times \log _{10}\left[\frac{(\mathrm{H} \gamma / \mathrm{H} \beta)_{\mathrm{obs}}}{(\mathrm{H} \gamma / \mathrm{H} \beta)_{\mathrm{int}}}\right]
$$

In both cases, the subscripts "obs" and "int" refer to the observed line ratios and the expected intrinsic ratios, 
Table 5

Ugriz Extinction Indices

\begin{tabular}{|c|c|c|c|c|c|c|c|c|}
\hline$T_{\text {eff }}$ & $\frac{E\left(\frac{E(u-g)}{E(44-55)}\right.}{\bar{c}(4)}$ & $\frac{E(g-r)}{E(44-55)}$ & 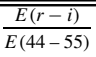 & $\frac{E(i-z)}{E(44-55)}$ & $\frac{E\left(\frac{E(u-g)}{E(44-55)}\right.}{E()^{\prime}}$ & $\frac{E(g-r)}{E(44-55)}$ & $\frac{E(r-i)}{E(44-55)}$ & $\frac{E(i-z)}{E(44-55)}$ \\
\hline & \multicolumn{8}{|c|}{$R(V)=2.5$} \\
\hline & \multicolumn{4}{|c|}{$E(44-55)=0.50$} & \multicolumn{4}{|c|}{$E(44-55)=1.00$} \\
\hline $7000 \mathrm{~K}$ & 1.101 & 1.000 & 0.562 & 0.433 & 1.114 & 0.979 & 0.560 & 0.433 \\
\hline $8000 \mathrm{~K}$ & 1.072 & 1.014 & 0.565 & 0.436 & 1.085 & 0.992 & 0.563 & 0.436 \\
\hline $9000 \mathrm{~K}$ & 1.054 & 1.028 & 0.567 & 0.438 & 1.067 & 1.006 & 0.565 & 0.438 \\
\hline $10000 \mathrm{~K}$ & 1.049 & 1.036 & 0.567 & 0.438 & 1.063 & 1.014 & 0.565 & 0.438 \\
\hline $12500 \mathrm{~K}$ & 1.068 & 1.045 & 0.567 & 0.437 & 1.082 & 1.022 & 0.565 & 0.436 \\
\hline $15000 \mathrm{~K}$ & 1.082 & 1.049 & 0.567 & 0.435 & 1.096 & 1.026 & 0.565 & 0.435 \\
\hline $20000 \mathrm{~K}$ & 1.094 & 1.055 & 0.567 & 0.434 & 1.108 & 1.032 & 0.565 & 0.434 \\
\hline $25000 \mathrm{~K}$ & 1.101 & 1.058 & 0.567 & 0.434 & 1.116 & 1.035 & 0.565 & 0.433 \\
\hline $30000 \mathrm{~K}$ & 1.104 & 1.062 & 0.567 & 0.433 & 1.118 & 1.038 & 0.565 & 0.433 \\
\hline \multirow[t]{2}{*}{$35000 \mathrm{~K}$} & 1.106 & 1.065 & 0.566 & 0.433 & 1.121 & 1.041 & 0.564 & 0.433 \\
\hline & \multicolumn{8}{|c|}{$R(V)=3.1$} \\
\hline
\end{tabular}

\begin{tabular}{|c|c|c|c|c|c|c|c|c|}
\hline $7000 \mathrm{~K}$ & \multicolumn{4}{|c|}{$E(44-55)=0.50$} & \multicolumn{4}{|c|}{$E(44-55)=1.00$} \\
\hline $8000 \mathrm{~K}$ & 1.059 & 1.051 & 0.625 & 0.506 & 1.073 & 1.031 & 0.623 & 0.506 \\
\hline $9000 \mathrm{~K}$ & 1.041 & 1.064 & 0.627 & 0.508 & 1.055 & 1.044 & 0.624 & 0.508 \\
\hline $12500 \mathrm{~K}$ & 1.053 & 1.081 & 0.627 & 0.506 & 1.068 & 1.060 & 0.624 & 0.506 \\
\hline $15000 \mathrm{~K}$ & 1.067 & 1.085 & 0.627 & 0.504 & 1.082 & 1.064 & 0.624 & 0.504 \\
\hline $20000 \mathrm{~K}$ & 1.078 & 1.091 & 0.627 & 0.503 & 1.094 & 1.069 & 0.624 & 0.503 \\
\hline \multirow[t]{2}{*}{$35000 \mathrm{~K}$} & 1.090 & 1.100 & 0.626 & 0.502 & 1.105 & 1.078 & 0.623 & 0.502 \\
\hline & \multicolumn{8}{|c|}{$R(V)=4.5$} \\
\hline
\end{tabular}

\begin{tabular}{|c|c|c|c|c|c|c|c|c|}
\hline \multirow[b]{2}{*}{$7000 \mathrm{~K}$} & \multicolumn{4}{|c|}{$E(44-55)=0.50$} & \multicolumn{4}{|c|}{$E(44-55)=1.00$} \\
\hline & 1.055 & 1.125 & 0.760 & 0.664 & 1.071 & 1.110 & 0.758 & 0.666 \\
\hline $8000 \mathrm{~K}$ & 1.028 & 1.137 & 0.764 & 0.668 & 1.043 & 1.121 & 0.761 & 0.670 \\
\hline $9000 \mathrm{~K}$ & 1.010 & 1.149 & 0.766 & 0.671 & 1.026 & 1.133 & 0.763 & 0.673 \\
\hline $10000 \mathrm{~K}$ & 1.005 & 1.157 & 0.766 & 0.671 & 1.021 & 1.141 & 0.763 & 0.673 \\
\hline $12500 \mathrm{~K}$ & 1.020 & 1.165 & 0.766 & 0.668 & 1.036 & 1.149 & 0.763 & 0.670 \\
\hline $15000 \mathrm{~K}$ & 1.032 & 1.169 & 0.766 & 0.666 & 1.049 & 1.152 & 0.763 & 0.667 \\
\hline $20000 \mathrm{~K}$ & 1.042 & 1.174 & 0.766 & 0.664 & 1.059 & 1.157 & 0.763 & 0.665 \\
\hline $25000 \mathrm{~K}$ & 1.048 & 1.177 & 0.766 & 0.662 & 1.065 & 1.160 & 0.763 & 0.664 \\
\hline $30000 \mathrm{~K}$ & 1.049 & 1.180 & 0.766 & 0.661 & 1.067 & 1.163 & 0.763 & 0.663 \\
\hline \multirow[t]{2}{*}{$35000 \mathrm{~K}$} & 1.051 & 1.184 & 0.764 & 0.662 & 1.069 & 1.166 & 0.761 & 0.663 \\
\hline & \multicolumn{8}{|c|}{$R(V)=6.0$} \\
\hline
\end{tabular}

\begin{tabular}{|c|c|c|c|c|c|c|c|c|}
\hline \multirow[b]{2}{*}{$7000 \mathrm{~K}$} & \multicolumn{4}{|c|}{$E(44-55)=0.50$} & \multicolumn{4}{|c|}{$E(44-55)=1.00$} \\
\hline & 1.021 & 1.220 & 0.909 & 0.838 & 1.038 & 1.211 & 0.906 & 0.843 \\
\hline $8000 \mathrm{~K}$ & 0.994 & 1.230 & 0.913 & 0.844 & 1.011 & 1.220 & 0.910 & 0.849 \\
\hline $9000 \mathrm{~K}$ & 0.977 & 1.241 & 0.915 & 0.847 & 0.994 & 1.231 & 0.912 & 0.852 \\
\hline $10000 \mathrm{~K}$ & 0.971 & 1.248 & 0.916 & 0.846 & 0.989 & 1.238 & 0.913 & 0.851 \\
\hline $12500 \mathrm{~K}$ & 0.984 & 1.256 & 0.915 & 0.842 & 1.002 & 1.245 & 0.912 & 0.847 \\
\hline $15000 \mathrm{~K}$ & 0.995 & 1.260 & 0.915 & 0.839 & 1.013 & 1.249 & 0.912 & 0.844 \\
\hline $20000 \mathrm{~K}$ & 1.003 & 1.265 & 0.915 & 0.837 & 1.021 & 1.253 & 0.912 & 0.841 \\
\hline $25000 \mathrm{~K}$ & 1.007 & 1.267 & 0.915 & 0.835 & 1.026 & 1.256 & 0.912 & 0.839 \\
\hline $30000 \mathrm{~K}$ & 1.008 & 1.270 & 0.915 & 0.834 & 1.027 & 1.259 & 0.912 & 0.838 \\
\hline $35000 \mathrm{~K}$ & 1.010 & 1.274 & 0.913 & 0.834 & 1.029 & 1.262 & 0.910 & 0.839 \\
\hline
\end{tabular}

respectively. Equations (10) and (11) show that the observed ratio is slightly sensitive to the value of $R$ for the $\mathrm{H} \alpha$ case, and not at all for the $\mathrm{H} \gamma$ case. The overall weakness of the $R$ dependence exists because the emission lines lie within or close to the region where the curves are normalized and, therefore, highly constrained.

For the case of the average Milky Way value of $R(55)=3.02$ (i.e., $R(V)=3.10$ ), the constant term in Equation (10) becomes
2.15. To find $E(B-V)$, this value is reduced by a factor of $\sim 0.99$ (see Table 4), yielding a scale factor of 2.13. This can be compared with the value of 1.97 as computed by Domínguez et al. (2013) using the attenuation law of Calzetti et al. (2000), which characterizes regions of starburst activity. Given the difference in the environments involved (Milky Way average versus starburst), this level of disagreement might be considered unsurprising. However, a closer look shows that it actually 
reveals a significant problem, namely that the Calzetti et al. (2000) law is not rigorously normalized to the $B V$ system. This is a common problem among most current representations of optical extinction and will be graphically illustrated in Section 5.1. In brief, the Calzetti et al. (2000) law is too steep in the optical and produces $E(B-V)$ values about $6 \%$ larger than intended. For example, if the curve were scaled to $E(B-V)=1.0$ and applied to an SED, synthetic photometry would reveal that the $(B-V)$ color of the SED actually increased by about 1.06 mag. A simple rescaling of the law in the optical, to force consistency with the intended normalization, eliminates most of the difference with our result for the $\mathrm{H} \alpha / \mathrm{H} \beta$ ratio.

For emission lines near the normalization region (4400-5500 $⿱$ ), the results for the average Milky Way curve, such as in Equations (10) and (11), are likely to be universally applicable. For emission lines shortward of the normalization region, however, the decrement method critically depends on the detailed nature of the extinction law (large- $R$, small- $R$, Milky Way average, starburst, and so on), because it is here that the wide range in normalized extinction curve shapes becomes evident.

\section{Discussion}

\subsection{Comparison to Previous Work}

Our new results are compared with past work in Figure 9. The left and right sides of the figure show the optical/NIR and UV spectral regions, respectively. The top panels on both sides show the extinction curves themselves, while the bottom panels highlight the differences between our results and those of the other investigations. Vertical offsets are used for clarity. Three representative values of $R(V)$ are illustrated (distinguished by color) for most of the comparison studies, which-with the exception of Bastiaansen (1992, "B92") in the optical and Fitzpatrick \& Massa (2007, "F07") in the UV-all present families of R-dependent curves. B92's and F07's results are explicit measures of mean diffuse medium extinction properties and are grouped with the $R(V)=3.1$ curves. The native units for all of the comparison studies are $k(\lambda-V)$, so we converted our $k(\lambda-55)$ curves to $k(\lambda-V)$ for this comparison, using Equation (4).

In the optical/NIR, the figure shows that the agreement among the various curves is at the level of $\sim 0.1$ within and slightly beyond the normalization region (i.e., $\sim 1.6-2.7 \mu \mathrm{m}^{-1}$ ). The work of B92 shows the best agreement with our results (for the case $R(V)=3.1$ ). B92's curve was produced using narrowband multicolor photometry, yielding a low-resolution estimate of the true wavelength dependence of the optical curve. The other studies, with the partial exception of Fitzpatrick (1999, "F99"), were not intended to yield detailed representations of the optical extinction law. Rather, they are generally "connect-the-dots" curves guided by the extinction ratios and effective wavelengths from broadband photometry. As such, they only crudely represent the actual shape of the curve, leading to the sizable deviations seen even within the normalization region. In general, these curves do not yield the intended values of $E(B-V), R(V)$, and other photometric extinction indices when applied to stellar SEDs, as already noted in the previous section for the Calzetti et al. (2000) starburst extinction curve (see the discussion in F99).

The discrepancies between the comparison curves and ours are larger outside the normalization region. None of the earlier studies utilize measurements in the gap between the optical and
UV, and the curves in these regions are extrapolations or interpolations between fundamentally different data sets. Note the large discrepancy in the F99 curve at long wavelengths and large $R(V)$. F99 used a simple interpolation between the optical (at $\sim 6000 \AA$ ) and the NIR $J H K$ region, and this clearly does not follow the true shape of the curve as revealed by the new spectrophotometry.

The right-hand panel of Figure 9 shows good agreement among all the studies on the properties of mean Milky Way extinction in the UV (i.e., for the case $R(V)=3.1$ ). This is not surprising because there is considerable overlap in the sight lines used in the various studies, all having been drawn from the database of $I U E$ satellite observations. The differences that do exist arise from a number of potential sources, including (1) the specific subset of sight lines used, (2) the use of updated photometry (particularly the 2MASS database), (3) differing techniques used to produce the curves (e.g., pair method versus "extinction without standards"), and (4) the technique used to derive the mean curve (e.g., via the $R(V)$-dependence or from a simple average). The differences among the various studies are much larger as $R(V)$ departs from the average Milky Way value. In the most general terms, our results show a weaker overall $R(V)$-dependence than most of the studies illustrated in Figure 9. Given that the $R(V)$-dependences are strongly driven by a relatively small number of sight lines at extreme $R(V)$, the specific sample selections are likely to have a major effect on the results. The other sources of uncertainty listed above for the mean curve are also probable contributors.

To address the sensitivity of our results to the sample selection, we reexamined the 328-star sample from F07. They derived a mean extinction curve from a simple average of all curves with $2.4 \leqslant R(V) \leqslant 3.6$, but did not quantify the $R(V)$ dependence. We used the same methodology as described in Section 4.1 to measure the UV $R(V)$-dependence of the 260 stars from F07 for which $E(B-V)>0.30$. The results are shown by the blue dotted curves in the three panels of Figure 6, converted to the $k(\lambda-55)$ normalization. As can be seen, the F07 sample yielded results that are very similar to those derived here. Specifically, the $R(55)=3.02$ curve and the sample standard deviation curve are nearly identical to the current results and virtually indistinguishable in Figure 6 (top and bottom panels), while the $R(V)$-dependence is similar in shape and differs by only $\sim 10 \%$ from the current result (middle panel of Figure 6). We conclude that the agreement between F07 and our much smaller current sample indicates that the new results are not subject to a strong sample bias and are generally applicable, at least to the extent that the current body of UV Milky Way extinction measurements can be considered representative of the Milky Way.

\subsection{Variations beyond R-dependence}

Our focus in this paper has been on the trends in the shapes of interstellar extinction curves that can be related to changes in $R$. While this accounts for the broadest trends seen among extinction curves (see Figure 8), it by no means reproduces all of the observed sight line-to-sight line variations. The $\sigma[k(\lambda-55)]$ curve in Figure 6 reveals that there is significant scatter around the mean $R$-dependence, which is typically much larger than the individual curve uncertainties (with the likely exception of the longest-wavelength G750L observations). This cosmic scatter reveals specific variations among the grain populations 

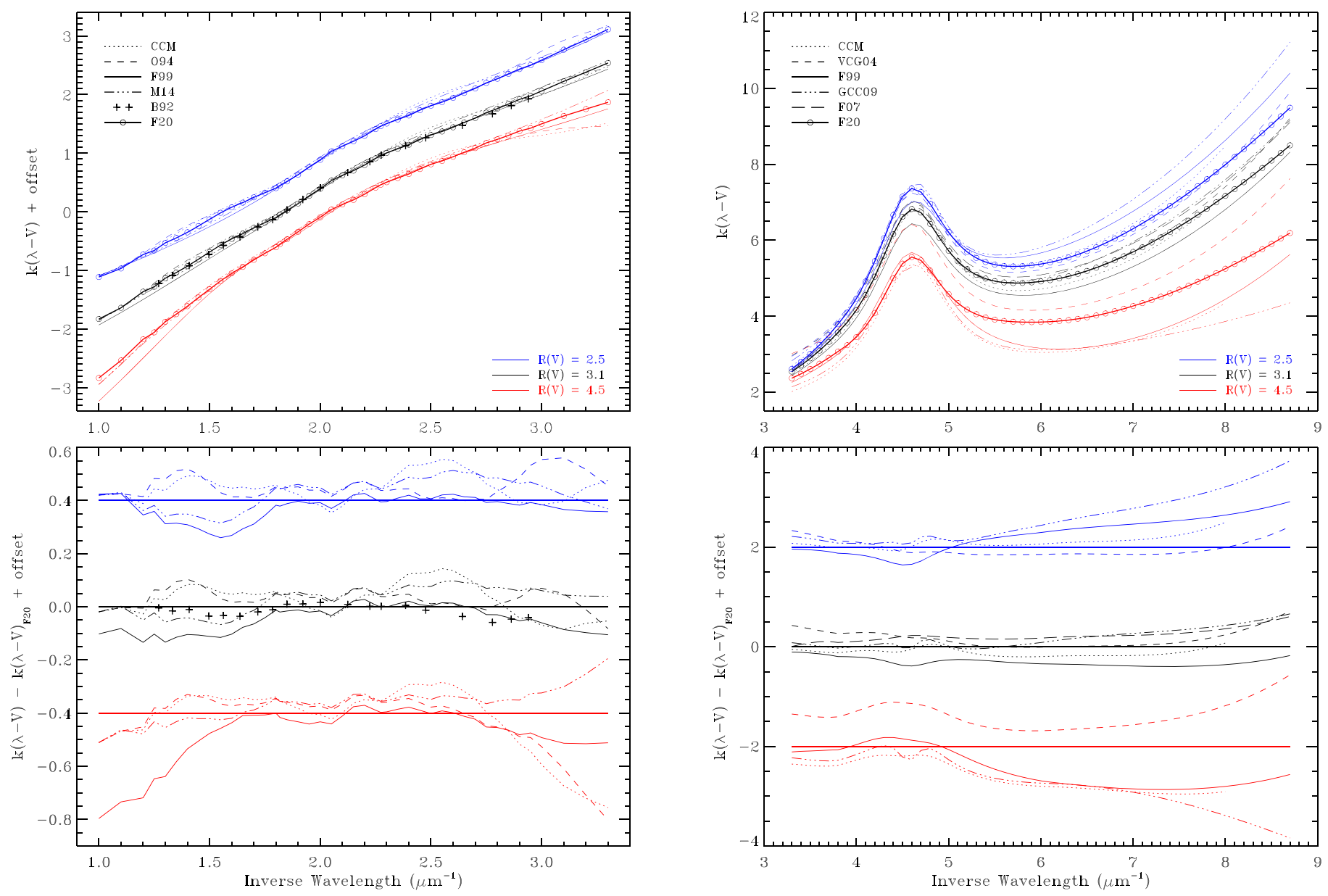

Figure 9. Comparison between our results and previously published estimates of extinction in the optical/NIR (left side) and UV (right side). The extinction curves themselves are shown in the upper half of each panel. The differences between these curves and our new results are shown in the lower half of each panel. Results for several values of $R(V)$ are presented and identified by different colors (see the lower right portion of each upper panel). The various published studies are distinguished by differing line styles and symbols, as indicated in the top left portion of each upper panel. Vertical offsets are utilized to increase clarity. The key to the various studies, in alphabetical order, is as follows: B92 = Bastiaansen (1992); CCM = Cardelli et al. (1989); F07 = Fitzpatrick \& Massa (2007); F20 = this paper; F99 = Fitzpatrick (1999); GCC09 = Gordon et al. (2009); M14 = Maíz Apellániz et al. (2014); O94 = O’Donnell (1994); VCG04 = Valencic et al. (2004).

that are not distinguished (or distinguishable) by $R$. Notably, the strength of the $2175 \AA$ bump and the level of the curve at the shortest UV wavelengths are only lightly tied to $R$. The level of the far-UV curve is particularly interesting. There are several sight lines (e.g., toward HD 204827, HD 210072, and HD 210121) that are well known for having very steep UV extinction curves, seemingly accompanied by weaker-than-average $2175 \AA$ A bumps (see F07). These are not reproduced by our $R$-dependent curves. In fact, these sight lines are among those that were "clipped" from the $R(55)$-dependence because they do not follow the general trend, as can be seen in the bottom panels of Figure 5. These relatively rare, steep far-UV/weak-bump sight lines appear to be associated in general with low values of $R$, but not in an easily predictable way. They may represent a separate $R$-dependent sequence of curves or perhaps demonstrate an extreme sensitivity of the curve to grain population details in environments where a generally small value of $R$ pertains. In either case, a detailed study of the grain environments along such sight lines may be necessary to understand the formation of such curves. Extinction studies in external galaxies, such as the Small Magellanic Cloud, where steep/weak-bumped curves appear to be the norm (e.g., Gordon et al. 2003), may also help shed light on the relationship between grain population characteristics and extinction curve properties.
An additional level of curve variability outside the scope of this paper is seen in the fine structure revealed by our measurements of optical extinction. As noted in Section 3.2 and illustrated in the curves of Figure 5, the optical region contains distinct extinction features known as the Very Broad Structure. A detailed examination of these features is presented by Massa et al. (2020). This study reveals significant sight lineto-sight line variations among these features, which are correlated with other aspects of the extinction curve, but not with $R$. See Massa et al. (2020) for the complete discussion.

\section{Summary}

We have produced the first spectrophotometrically derived measurement of the average Milky Way interstellar extinction curve over the wavelength range 1150 to $10000 \AA$ and determined its general dependence on the ratio of total-to-selective extinction in the optical, $R$, over the range $2.5 \leqslant R \leqslant 6.0$. This curve resolves structures in the optical spectral region down to a level of $\sim 100-200 \AA$ and fully resolves all structure in the UV region.

The curve is based on a unique, homogeneous data set consisting of IUE and HST/STIS spectrophotometry of normal, early-type stars. These data were all calibrated in a mutually consistent way and fit to model atmospheres to produce a 
uniform, self-consistent set of extinction curves that span 1150 to $10000 \AA$ at high resolution and extended to $2.2 \mu \mathrm{m}$ with broadband $J H K$ photometry. These curves provide the first systematic measurements of extinction in the transition region between past ground-based studies and space-based UV measurements. The use of optical spectrophotometry allowed us to eliminate the uncertainties introduced by normalizing extinction curves by broadband optical photometry, and we adopted a monochromatic normalization scheme, based on the extinction at 4400 and $5500 \AA$. The new curves were used to evaluate the dependence of extinction on $R$ over the full wavelength range, and we ultimately parameterized this dependence as a simple linear function of $R$, essentially a first-order Taylor expansion. The average Milky Way extinction curve we present is that which corresponds to the case $R(V)=3.10$. We present this curve in tabular form, along with the linear $R$ dependence and a measure of the curve-to-curve variations about this mean dependence.

Because the coverage between 1150 and $10000 \AA$ is free of gaps and at relatively high resolution, it is possible to use the tabular data to derive the behavior of extinction for any given photometric system, and several examples are presented. The new, high-resolution curves also allow the study of extinction features on intermediate wavelength scales in the optical. While such features have been seen before, the new curves show them with considerably more detail and higher $\mathrm{S} / \mathrm{N}$ than in the past. The exact shape and sight line-to-sight line variability of this structure are discussed more thoroughly by Massa et al. (2020).

E.F. and D.M. acknowledge support by grant No. HST-GO13760 provided by NASA through the Space Telescope Science Institute, which is operated by the Association of Universities for Research in Astronomy, Inc., under NASA contract NAS5-26555. Some of the data presented in this paper were obtained from the Mikulski Archive for Space Telescopes. STScI is operated by the Association of Universities for Research in Astronomy, Inc., under NASA contract NAS526555 .

\section{Appendix A STIS Data Reduction}

The G430L and G750L spectra are processed with the IDT pipeline software written in IDL by D.J. Lindler in 1996-1997. This IDL data reduction is more flexible and offers the following advantages over the STScI pipeline results that are available from the MAST $^{11}$ archive:

1. Small shifts in pointing or instrumental flexure between the images of a cosmic-ray split (CR-split) observation can often cause an erroneous reduction of net signal, because pixels with the lower signal are retained and the higher pixel value is rejected in the case that the CR-split image pair is not perfectly aligned and pixel pair values differ by more than their statistical noise estimates. Figure 10 shows a standard display of the IDT processing, which makes obvious the deficiency in the reduction with the default mult_noise $=0$ parameter for combining the CR-split pair of images that have a small shift. This excess of false CR flags is completely eliminated by a relaxed mult_noise $=0.04$ value, which does not significantly increase the number of

\footnotetext{
11 http://archive.stsci.edu/hst/search.php
}

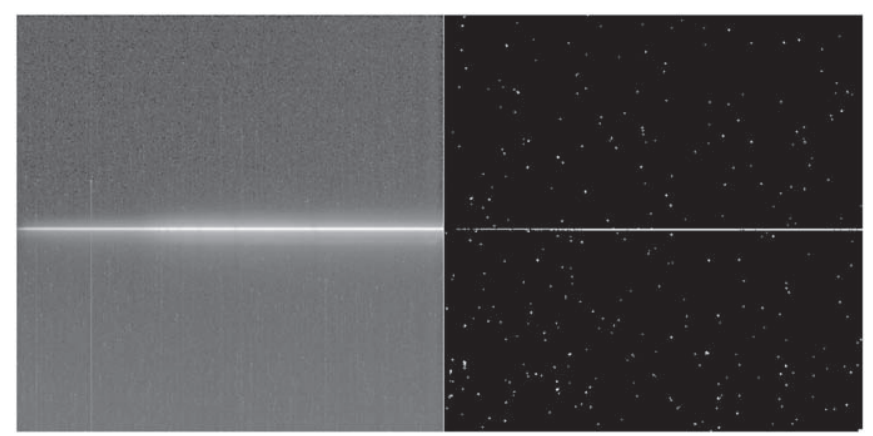

Figure 10. $1024 \times 1024$ pixel STIS CCD image of the G430L CR-split $=2$ observation of HD146285 (ocmv0j010) on the left and the corresponding image on the right, which flags the location of each falsely detected cosmic-ray hit for the default mult_noise $=0$. Because of a pointing shift between the pair of CR-split images, there is an easily recognized excess of flagged pixels at the location of the spectrum. With a relaxed value of mult_noise $=0.04$, there is no excess of CR-flagged pixels at the spectral location, and the correct combined image of the CR-split pair is produced.

unflagged actual cosmic-ray hits because of our relatively short exposure times. Figure 11 shows the error in extracted spectral signal for mult_noise $=0$ in black and the corresponding error in the STScI pipeline error of the ocmv0j010_sx1.fits reduction in red. Errors in spectral shape of $2 \%$ are common and have two narrower band error features in the STScI standard product. For our observed 98 SNAP program stars, 12 G430L and seven G750L observations required relaxed values for mult_noise in order to extract the proper spectral signal. For slightly saturated STIS spectral observations, the CR-reject algorithm also fails with a display similar to Figure 10. However, changing mult_noise has little effect. Because saturated data at Gain $=4$ do not lose charge and the total signal is still present in the raw images, the solution for extracting the proper spectrum is to omit any CR-reject attempts and average the extracted signal from the pair of *flt.fits images. This approach works well in the common case of a bright star and short exposures where cosmic-ray hits are minimal.

2. For G750L, especially at the longer wavelengths, the spectral width has increased significantly in recent years, and a wider extraction height (EXTRSIZE) of 11 pixels, instead of the STScI pipeline EXTRSIZE $=7$ pixels, is required to maintain photometric repeatability of G750L observations (Bohlin \& Proffitt 2015). The EXTRSIZE $=7$ spectra show errors in spectral shape of up to $3 \%$ in ratio of the long to the short wavelengths of G750L. The wider extraction width encompasses more low signal pixel values, which decreases the spectral S/N slightly.

3. Pixels that are flagged as hot are not used, and those values are reset to the value interpolated in the spectral direction of the affected row of the image before extraction of the spectrum. Very occasionally, a hot pixel flag can occur at the center of a spectral line, resulting in a small reduction in equivalent width. For this paper, our interest is in the continuum and not in precise equivalentwidth measures.

4. To remove the CCD fringing in G750L, tungsten lamp spectra through the 0 " $03 \times 0$." 09 slit must be used to align this artificial star with the position of the actual star. The following spline nodes for fitting these tungsten flats are a prerequisite for the G750L sensitivity calibration: 5240, 5265, 5300, 5318, 5340, 5380, 5420, 5460, 5500, 


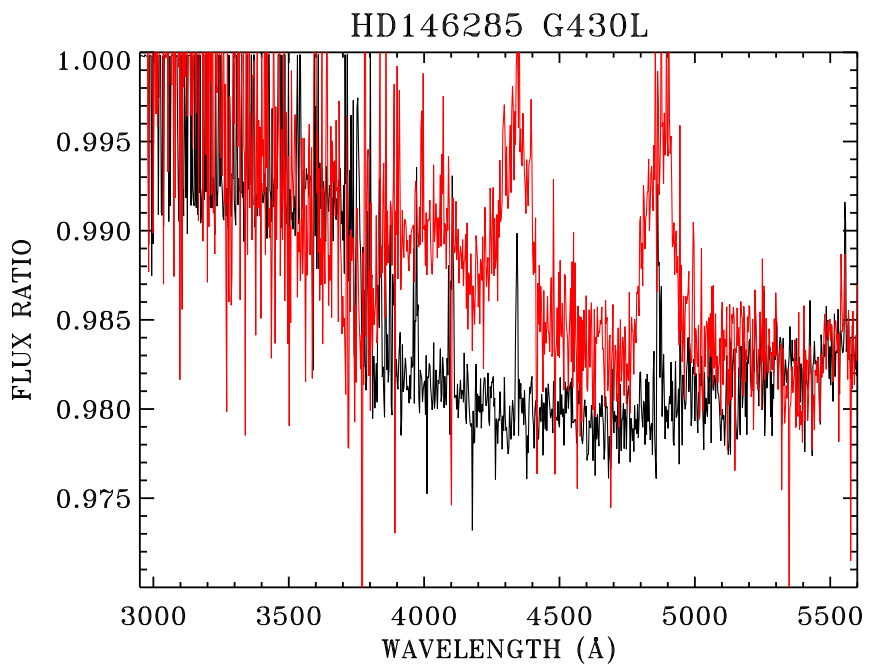

Figure 11. Ratio (black) of extracted spectra for mult_noise $=0$ to mult noise $=0.04$ for the CR-split $=2$ observation ocmv0j010 and the ratio (red) of the STScI pipeline reduction ocmv0j010_sx1.fits to the same denominator with the IDL parameter mult_noise $=0.04$. Errors in spectral shape of up to $\sim 2 \%$ exist for the two incorrect spectral extractions.

$5580,5700,5800,5840,6000,6200,6300,6450,6550$, $6600,6620,6700,6800,6900,7000,7150,7400,7600$, $7800,8000,8300,8500,9100,9500,9800$, and $10200 \AA$. These wavelengths are selected to track the features of the tungsten lamp spectrum, while maintaining the proper stiffness and avoiding $\sim 1 \%$ bumps due to the $\sim 20 \%$ fringe structure at the longer wavelengths. The nodes below $6600 \AA$ are not crucial because the fringe flat is set to unity below $6620 \AA$. These short-slit tungsten flats are applied to all G750L observations after normalization to unity, reduction of the fringe amplitude by $11 \%$ to compensate for narrower PSF of the artificial star, and cross-correlating with the stellar spectrum to remove small residual offsets in the dispersion direction. An example of a fringe flat for ocmv0j020 appears in Figure 12.

In addition to the above data extraction advantages, our postprocessing includes the CTE corrections of Goudfrooij et al. (2006) and an up-to-date correction for the changes in STIS sensitivity with time and with temperature. The observations of the three primary flux standards G191B2B, GD153, and GD71, as corrected for CTE and the latest measures of sensitivity change with time, produce a current absolute flux calibration (CALSPEC; see Bohlin et al. 2014).

\section{Appendix B}

\section{Conversions between Extinction Relationship Equations}

Interstellar extinction curves are commonly expressed using the " $k$ " notation, in the form

$$
k(\lambda-V) \equiv \frac{E(\lambda-V)}{E(B-V)} \equiv \frac{A(\lambda)-A(V)}{A(B)-A(V)},
$$

where $A(\lambda)$ is the total extinction at wavelength $\lambda$, and $B$ and $V$ refer to measurements made using the Johnson $B$ and $V$ filters. A related quantity, commonly known as $R$, is the ratio of

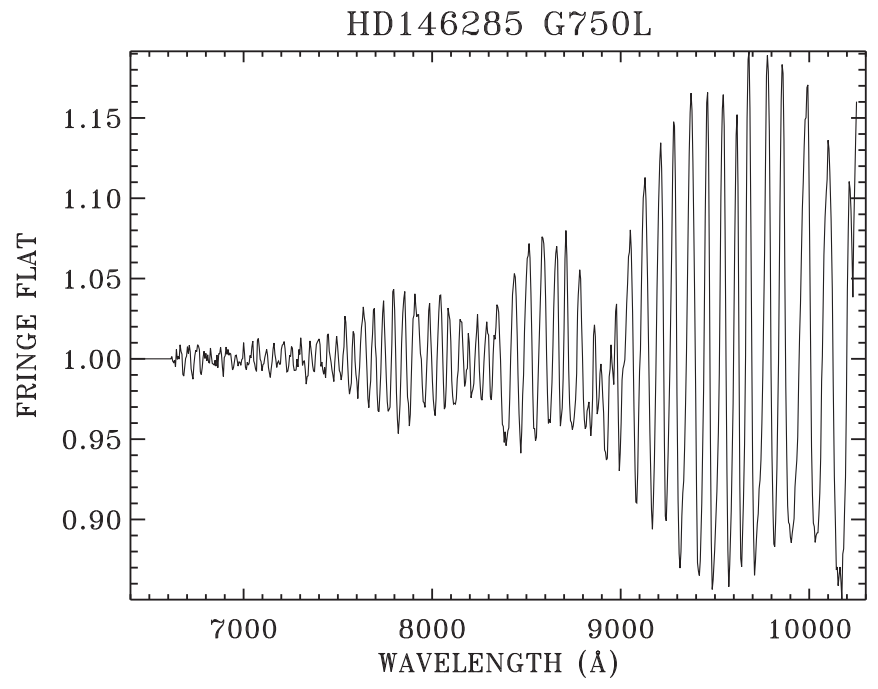

Figure 12. Fringe flat correction for the G750L spectrum ocmv0j020 of HD146285.

total-to-selective extinction in the optical, given by

$$
R(V) \equiv \frac{A(V)}{E(B-V)} .
$$

In this paper, we adopt the analogous forms

$$
k(\lambda-55) \equiv \frac{E(\lambda-55)}{E(44-55)} \equiv \frac{A(\lambda)-A(55)}{A(44)-A(55)}
$$

and

$$
R(55) \equiv \frac{A(55)}{E(44-55)},
$$

in which measurements with the Johnson $B$ and $V$ filters are replaced by monochromatic measurements at 4400 and $5500 \AA$, respectively. Our data show that the two normalizations are related by

$$
k(\lambda-55)=\alpha k(\lambda-V)+\beta,
$$

which implies

$$
R(55)=\alpha R(V)-\beta .
$$

The precise values of $\alpha$ and $\beta$ depend on the shapes of the stellar energy distributions (reddened and unreddened), due to the broadband nature of the $B$ and $V$ filters. For our particular sample, which on average consists of middle B stars with $E(B-V) \simeq 0.5$, we find that $\alpha=0.990$ and $\beta=0.049$. With these coefficients, the generally accepted Milky Way mean value of $R(V)_{0}=3.10$ corresponds to $R(55)_{0}=3.02$.

As described in the text, we find the following functional relationship between the $k(\lambda-55)$ extinction curves and $R(55)$ :

$$
k(\lambda-55)=s(\lambda-55)\left[R(55)-R(55)_{0}\right]+k(\lambda-55)_{0},
$$

which is essentially a first-order Taylor expansion in $R(55)$ at each wavelength. In this equation, $s(\lambda-55)$ is the observed slope between $R(55)$ and $k(\lambda-55), R(55)_{0}$ is the average Milky Way value of $R(55)$, and $k(\lambda-55)_{0}$ is the extinction curve corresponding to $R(55)_{0}$. The complete sets of $k(\lambda-55)_{0}$ and $s(\lambda-55)$ values, which allow a detailed extinction curve 
to be constructed over the range 1150 to $10000 \AA$, are given in Table 3.

In their early investigation of the $R$-dependence of extinction, CCM adopted a different extinction curve normalization, that is, $A(\lambda) / A(V)$. From Equations (12) and (13), it can be seen that this is related to $k(\lambda-\mathrm{V})$ by

$$
\frac{A(\lambda)}{A(V)}=\frac{k(\lambda-V)}{R(V)}+1 \text {. }
$$

CCM parameterized the $R$-dependence of $A(\lambda) / A(V)$ as

$$
\frac{A(\lambda)}{A(V)}=a(\lambda)+b(\lambda) R(V)^{-1} .
$$

At first look, this appears to be a very different functional relationship between $R$ and the shape of the extinction curve than we have found (i.e., Equation (18)). However, when Equation (19) is substituted into Equation (20), the following result is obtained:

$$
k(\lambda-V)=[a(\lambda)-1] R(V)+b(\lambda) .
$$

Thus it is seen that, like us, CCM found their extinction curves to be linearly related to $R$ when expressed in the " $k$ " notation. Moreover, the relationship between CCM's $a(\lambda)$ and $b(\lambda)$ and our coefficients $s(\lambda-55)$ and $k(\lambda-55)_{0}$ can be found by comparing Equations (18) and (21) and making the appropriate substitutions from Equations (16) and (17). The result is

$$
\begin{aligned}
& a(\lambda)=s(\lambda-55)+1 \\
& b(\lambda)=-s(\lambda-55)\left[\frac{R(55)_{0}+\beta}{\alpha}\right]+\left[\frac{k(\lambda-55)_{0}-\beta}{\alpha}\right] .
\end{aligned}
$$

In principle, identical results would be achieved regardless of whether our " $k$ " normalization or CCM's $A(\lambda)$ normalization was used. One must keep in mind, however, that these mathematical transformations only apply in the absence of errors. Least-squares solutions resulting from the two different formulations could yield different results.

\section{ORCID iDs}

E. L. Fitzpatrick (1) https://orcid.org/0000-0002-2371-5477 Derck Massa (ㄴ) https://orcid.org/0000-0002-9139-2964 Karl D. Gordon (1) https://orcid.org/0000-0001-5340-6774 Ralph Bohlin (10 https://orcid.org/0000-0001-9806-0551 Geoffrey C. Clayton (1) https://orcid.org/0000-0002-0141-7436

\section{References}

Aiello, S., Barsella, B., Chlewicki, G., et al. 1988, A\&AS, 73, 195 Aiello, S., Barsella, B., Guidi, I., Penco, U., \& Perinotto, M. 1982, Ap\&SS, 87, 463

Bastiaansen, P. A. 1992, A\&AS, 93, 449

Boggs, D., \& Bohm-Vitense, E. 1989, ApJ, 339, 209

Bohlin, R. C., Gordon, K. D., \& Tremblay, P. E. 2014, PASP, 126, 711

Bohlin, R. C., Mészáros, S., Fleming, S. W., et al. 2017, AJ, 153, 234

Bohlin, R. C., \& Proffitt, C. R. 2015, Instrument Science Report STIS 2015-01 (v1), 1

Borgman, J. 1960, BAN, 15, 255

Bostroem, K. A., \& Proffitt, C. 2011, STIS Data Handbook v. 6.0 (Baltimore, MD: STScI)

Bouigue, M. R. 1959, POHP, 4, 52

Boulon, J., Duflot, M., \& Fehrenbach, C. 1958, JO, 42, 1

Calzetti, D., Armus, L., Bohlin, R. C., et al. 2000, ApJ, 533, 682
Calzetti, D., Kinney, A. L., \& Storchi-Bergmann, T. 1994, ApJ, 429, 582

Cardelli, J. A., Clayton, G. C., \& Mathis, J. S. 1989, ApJ, 345, 245

Casagrande, L., \& VandenBerg, D. A. 2014, MNRAS, 444, 392

Clayton, G. C., \& Fitzpatrick, E. L. 1987, AJ, 93, 157

Clayton, G. C., Gordon, K. D., \& Wolff, M. J. 2000, ApJS, 129, 147

Clayton, G. C., Wolff, M. J., Sofia, U. J., Gordon, K. D., \& Misselt, K. A. 2003, ApJ, 588, 871

Cohen, M., Walker, R. G., Barlow, M. J., \& Deacon, J. R. 1992, AJ, 104, 1650

Cohen, M., Wheaton, W. A., \& Megeath, S. T. 2003, AJ, 126, 1090

Cowley, A., Cowley, C., Jaschek, M., \& Jaschek, C. 1969, AJ, 74, 375

Crawford, D. L. 1975, PASP, 87, 481

Divan, L. 1954, AnAp, 17, 456

Doi, M., Tanaka, M., Fukugita, M., et al. 2010, AJ, 139, 1628

Domínguez, A., Siana, B., Henry, A. L., et al. 2013, ApJ, 763, 145

Feast, M. W., Thackeray, A. D., \& Wesselink, A. J. 1955, MmRAS, 67, 51

Feast, M. W., Thackeray, A. D., \& Wesselink, A. J. 1957, MmRAS, 68, 1

Fitzgerald, M. P. 1970, A\&A, 4, 234

Fitzpatrick, E. L. 1999, PASP, 111, 63

Fitzpatrick, E. L. 2004, in ASP Conf. Ser. 309, Astrophysics of Dust, ed A. N. Witt, G. C. Clayton, \& B. T. Draine (San Francisco, CA: ASP), 33

Fitzpatrick, E. L., \& Massa, D. 1986, ApJ, 307, 286

Fitzpatrick, E. L., \& Massa, D. 1988, ApJ, 328, 734

Fitzpatrick, E. L., \& Massa, D. 1990, ApJS, 72, 163

Fitzpatrick, E. L., \& Massa, D. 2005a, AJ, 130, 1127

Fitzpatrick, E. L., \& Massa, D. 2005b, AJ, 129, 1642

Fitzpatrick, E. L., \& Massa, D. 2007, ApJ, 663, 320

Gallagher, J. S., III, Hunter, D. A., \& Tutukov, A. V. 1984, ApJ, 284, 544

Garrison, R. F. 1967, ApJ, 147, 1003

Garrison, R. F., \& Kormendy, J. 1976, PASP, 88, 865

Gordon, K. D., Cartledge, S., \& Clayton, G. C. 2009, ApJ, 705, 1320

Gordon, K. D., Clayton, G. C., Misselt, K. A., Land olt, A. U., \& Wolff, M. J. 2003, ApJ, 594, 279

Goudfrooij, P., Maíz Apellániz, J., Brown, T., \& Kimble, R. 2006, Instrument Science Report STIS 2006-01

Gray, R. O., \& Corbally, C. J. 1994, AJ, 107, 742

Grevesse, N., Asplund, M., Sauval, A. J., \& Scott, P. 2013, in ASP Conf. Ser. 479, Progress in Physics of the Sun and Stars: A New Era in Helio- and Asteroseismology, ed. H. Shibahashi \& A. E. Lynas-Gray (San Francisco, CA: ASP), 481

Grevesse, N., \& Sauval, A. J. 1998, SSRv, 85, 161

Guetter, H. H. 1968, PASP, 80, 197

Hackwell, J. A., Hecht, J. H., \& Tapia, M. 1991, ApJ, 375, 163

Herbig, G. H. 1975, ApJ, 196, 129

Hill, P. W. 1970, MNRAS, 150, 23

Hiltner, W. A. 1956, ApJS, 2, 389

Hoffleit, D. 1956, ApJ, 124, 61

Houk, N. 1978, Michigan Catalogue of Two-dimensional Spectral Types for the HD Stars (Ann Arbor, MI: Univ. Michigan)

Houk, N., \& Cowley, A. P. 1975, University of Michigan Catalogue of twodimensional Spectral Types for the HD Stars, Vol. I. Declinations -90 to -53 (Ann Arbor, MI: Univ. Michigan)

Johnson, H. L. 1962, ApJ, 136, 1135

Johnson, H. L., \& Borgman, J. 1963, BAN, 17, 115

Johnson, H. L., \& Morgan, W. W. 1953, ApJ, 117, 313

Johnson, H. L., \& Morgan, W. W. 1955, ApJ, 122, 429

Kennicutt, R. C., Jr. 1983, ApJ, 272, 54

Kurucz, R. L. 1991, in Stellar Atmospheres: Beyond Classical Models, ed. L. Crivellari, I. Hubeny, \& D. G. Hummer (Dordrecht: Reidel), 441

Lanz, T., \& Hubeny, I. 2003, ApJS, 146, 417

Lanz, T., \& Hubeny, I. 2007, ApJS, 169, 83

Maíz Apellániz, J. 2007, in ASP Conf. Ser. 364, The Future of Photometric, Spectrophotometric and Polarimetric Standardization, ed. C. Sterken (San Francisco, CA: ASP), 227

Maíz Apellániz, J., Evans, C. J., Barbá, R. H., et al. 2014, A\&A, 564, A63

Maíz Apellániz, J., \& Pantaleoni González, M. 2018, A\&A, 616, L7

Maíz-Apellániz, J., Walborn, N. R., Galué, H. Á., \& Wei, L. H. 2004, ApJS, 151,103

Massa, D., \& Fitzpatrick, E. L. 2000, ApJS, 126, 517

Massa, D., Fitzpatrick, E. L., \& Gordon, K. D. 2020, ApJ, submitted

Massa, D., Savage, B. D., \& Fitzpatrick, E. L. 1983, ApJ, 266, 662

Massey, P., \& Johnson, J. 1993, AJ, 105, 980

Mathis, J. S., \& Cardelli, J. A. 1992, ApJ, 398, 610

Meadows, A. J. 1961, MNRAS, 123, 81

Metreveli, M. D. 1968, AbaOB, 38, 93

Morgan, W. W., Abt, H. A., \& Tapscott, J. W. 1978, Revised MK Spectral Atlas for Stars Earlier than the Sun (Williams Bay, WI: Yerkes Observatory) 
Morgan, W. W., Code, A. D., \& Whitford, A. E. 1955, ApJS, 2, 41

Morgan, W. W., Whitford, A. E., \& Code, A. D. 1953, ApJ, 118, 318

Morrell, N., Garcia, B., \& Levato, H. 1988, PASP, 100, 1431

Muzzio, J. C., \& Rydgren, A. E. 1974, AJ, 79, 864

O’Donnell, J. E. 1994, ApJ, 422, 158

Osawa, K. 1959, ApJ, 130, 159

Panek, R. J. 1983, ApJ, 270, 169

Racine, R. 1968a, AJ, 73, 588

Racine, R. 1968b, AJ, 73, 233

Riley, A. 2019, STIS Instrument Handbook v. 18.0 (Baltimore MD: STScI)

Roman, N. G. 1955, ApJS, 2, 195

Schild, R., Neugebauer, G., \& Westphal, J. A. 1971, AJ, 76, 237

Schild, R. E. 1965, ApJ, 142, 979

Schild, R. E., \& Chaffee, F. 1971, ApJ, 169, 529
Slettebak, A. 1968, ApJ, 154, 933

Smartt, S. J., Dufton, P. L., \& Rolleston, W. R. J. 1996, A\&A, 305, 164 Smith, M. A. 1972, ApJ, 175, 765

Turner, D. G., Grieve, G. R., Herbst, W., \& Harris, W. E. 1980, AJ, 85, 1193

Valencic, L. A., Clayton, G. C., \& Gordon, K. D. 2004, ApJ, 616, 912

van Leeuwen, F. 2007, A\&A, 474, 653

Vrba, F. J., \& Rydgren, A. E. 1984, ApJ, 283, 123

Walborn, N. R. 1971, ApJS, 23, 257

Weingartner, J. C., \& Draine, B. T. 2001, ApJ, 548, 296

Whiteoak, J. B. 1966, ApJ, 144, 305

Whitford, A. E. 1958, AJ, 63, 201

Witt, A. N., Bohlin, R. C., \& Stecher, T. P. 1984, ApJ, 279, 698

York, D. G. 1971, ApJ, 166, 65

Zubko, V., Dwek, E., \& Arendt, R. G. 2004, ApJS, 152, 211 